\title{
Global Aeroheating Measurements of Shock-Shock Interactions on a Swept Cylinder
}

\author{
Michelle L. Mason ${ }^{1}$, Scott A. Berry ${ }^{2}$ \\ NASA Langley Research Center, Hampton, VA, 23681
}

\begin{abstract}
The effects of fin leading-edge radius and sweep angle on peak heating rates due to shock-shock interactions were investigated in the NASA Langley Research Center 20-Inch Mach 6 Air Tunnel. The cylindrical leading-edge fin models, with radii varied from 0.25 to 0.75 inches, represent wings or struts on hypersonic vehicles. A $9^{\circ}$ wedge generated a planar oblique shock at $16.7^{\circ}$ to the flow that intersected the fin bow shock, producing a shockshock interaction that impinged on the fin leading edge. The fin sweep angle was varied from $0^{\circ}$ (normal to the free-stream) to $15^{\circ}$ and $25^{\circ}$ swept forward. These cases were chosen to explore three characterized shock-shock interaction types. Global temperature data were obtained from the surface of the fused silica fins using phosphor thermography. Metal oil flow models with the same geometries as the fused silica models were used to visualize the streamline patterns for each angle of attack. High-speed zoom-schlieren videos were recorded to show the features and any temporal unsteadiness of the shock-shock interactions. The temperature data were analyzed using a one-dimensional semi-infinite method, as well as one- and two-dimensional finite-volume methods. These results were compared to determine the proper heat transfer analysis approach to minimize errors from lateral heat conduction due to the presence of strong surface temperature gradients induced by the shock interactions. The general trends in the leading-edge heat transfer behavior were similar for each explored shock-shock interaction type regardless of the leading-edge radius. However, the dimensional peak heat transfer coefficient augmentation increased with decreasing leading-edge radius. The dimensional peak heat transfer output from the two-dimensional code was about $20 \%$ higher than the value from a standard, semi-infinite one-dimensional method.
\end{abstract}

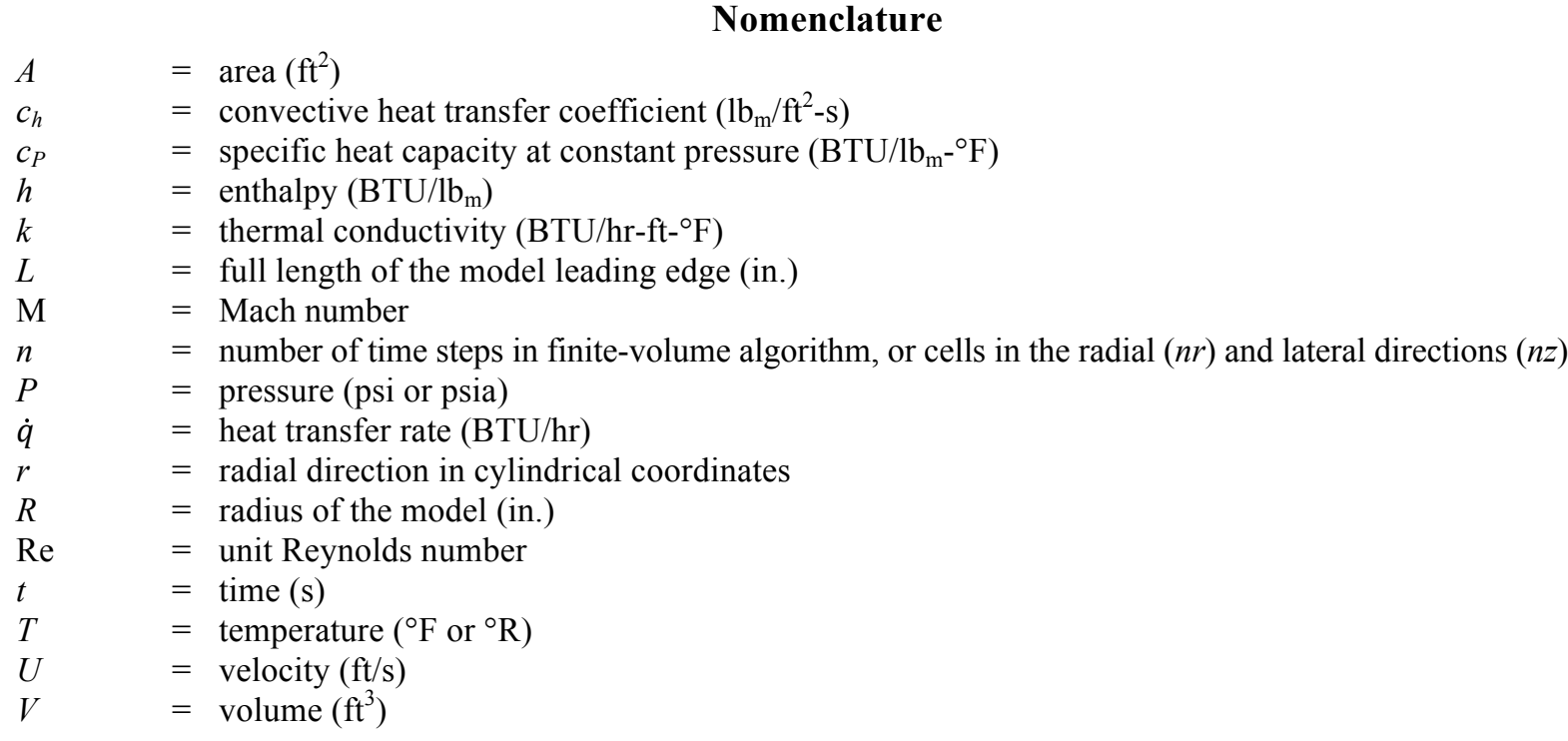

\footnotetext{
${ }^{1}$ Aerospace Engineer, Aerothermodynamics Branch, MS 408A, AIAA Member

${ }^{2}$ Aerospace Engineer, Aerothermodynamics Branch, MS 408A, AIAA Associate Fellow
} 


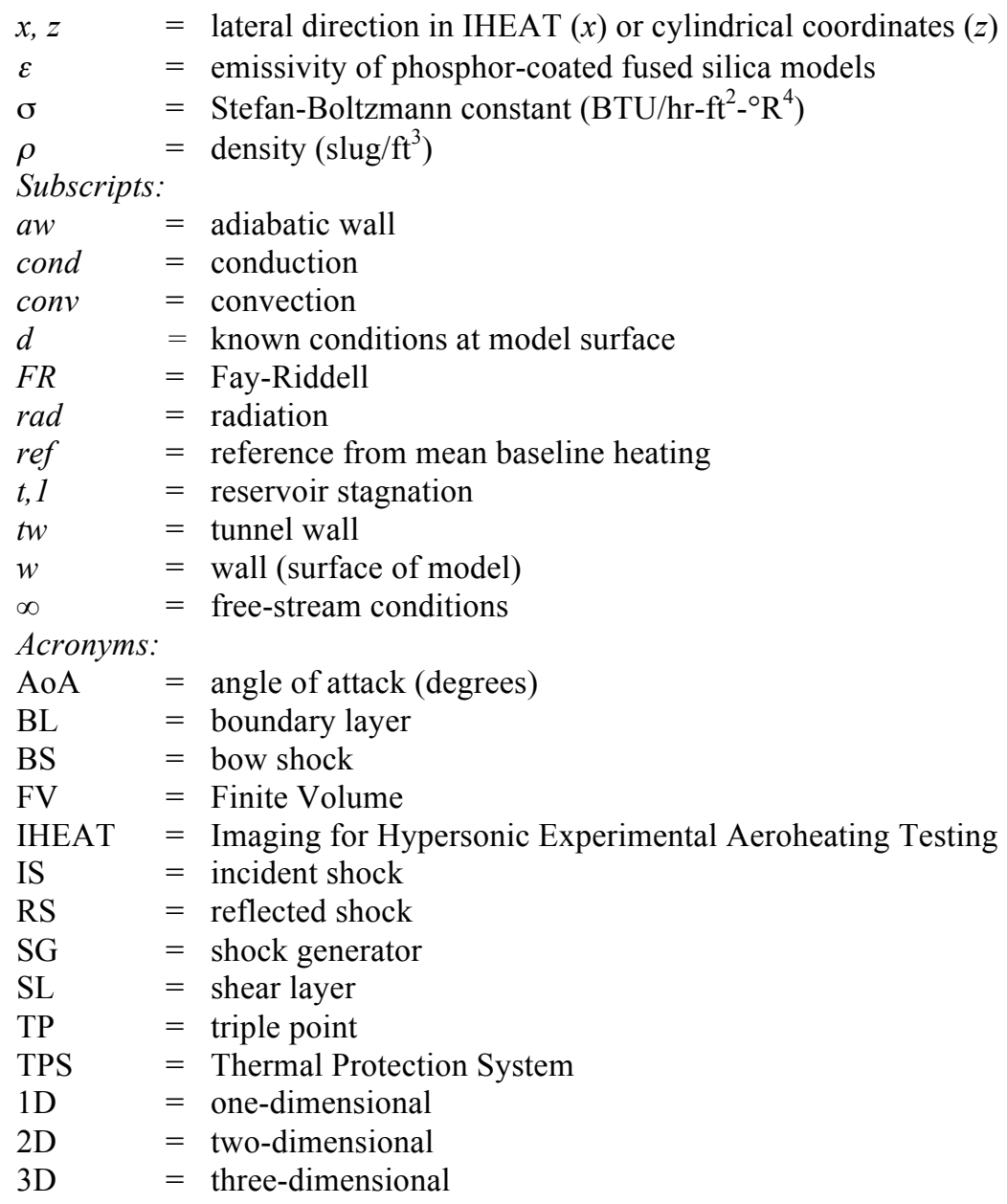

\section{Introduction}

Commercial, government and military applications rely on research into safe, reliable hypersonic technology. Vehicles designed to fly at hypersonic speeds, such as the now retired Space Shuttle Orbiter and either planes or missiles with integrated ramjet or supersonic combustion ramjet (scramjet) engines, can be subjected to a phenomenon called a shock-shock interaction that causes significant, localized surface temperature and pressure augmentations. ${ }^{1,2}$ Interactions between the vehicle's bow shock and the shock around a strut or a wing leading edge can compromise the vehicle's structural components in the absence of protective measures. Numerous experiments have investigated shock-shock interaction behavior and the resulting heating effects that occur in the hypersonic flight regime to aid in the development of Thermal Protection Systems (TPS). ${ }^{3-10}$ These studies helped classify shock-shock interaction types and pointed to the need for improved spatial resolution of the data in the regions affected by the interactions, as well as the potential need to add two-dimensional (2D) heat transfer analyses to the standard one-dimensional (1D) techniques if a strong lateral temperature gradient exists. Measurement techniques have gradually improved to provide better spatial resolution in shock-shock interaction heat transfer analyses.

Shock-shock interactions in hypersonic flow, as described in this study, involve an oblique incident shock generated by a flat plate shock generator (SG) that intersects a bow shock around a blunt body. Edney ${ }^{1}$ identified six general types of interactions between bow shocks around blunt bodies and incident shocks. The relative angle between the incident shock and the bow shock, as well as the strengths of these two shocks, dictate the features of the resulting shock impingement, such as the angle of the reflected shock, the number of shear layers that form, or the presence of a supersonic jet. These shock-shock interactions are sketched in Fig. 1. Of the six types Edney described, the Type III and IV interactions are likely to produce the worst heating to the vehicle since the shear layer or jet impinges directly on the surface. Since the time of Edney's study, another sub-type has been added to the shock-shock interaction lexicon, the Type IVa interaction, in which the supersonic jet is present but largely misses the surface of the body. 
Berry and Nowak ${ }^{11}$ experimentally investigated the effect of fin sweep angle on the increase in the peak heating on a leading edge or strut of a hypersonic vehicle due to different types of three-dimensional (3D) shock-shock interactions. This study included a systematic sweep of fin angles to capture most of the general classification types, as shown in the schlieren images in Fig. 1. Temperatures measured using Macor ${ }^{\circledR}$ models instrumented with thinfilm gages were reduced using a 1D heat transfer code. Berry and Nowak postulate that the non-dimensional peak heating increase for a Type IV interaction (on a 0.25 -in. radius model) might grow from nearly seven times the baseline value to a factor of ten if lateral conduction effects were considered in the heat transfer analysis. The peak heating increase for a Type III interaction was again nearly seven times the baseline value. Most of the wind tunnel runs in Ref. 11 were conducted at a unit Reynolds $\left(\mathrm{Re}_{\infty}\right)$ number of $2.1 \times 10^{6} / \mathrm{ft}$ with the SG at $9^{\circ}$ to the flow. The results of Ref. 11 were used to select a smaller subset of fin angles to investigate in the present study.

a)
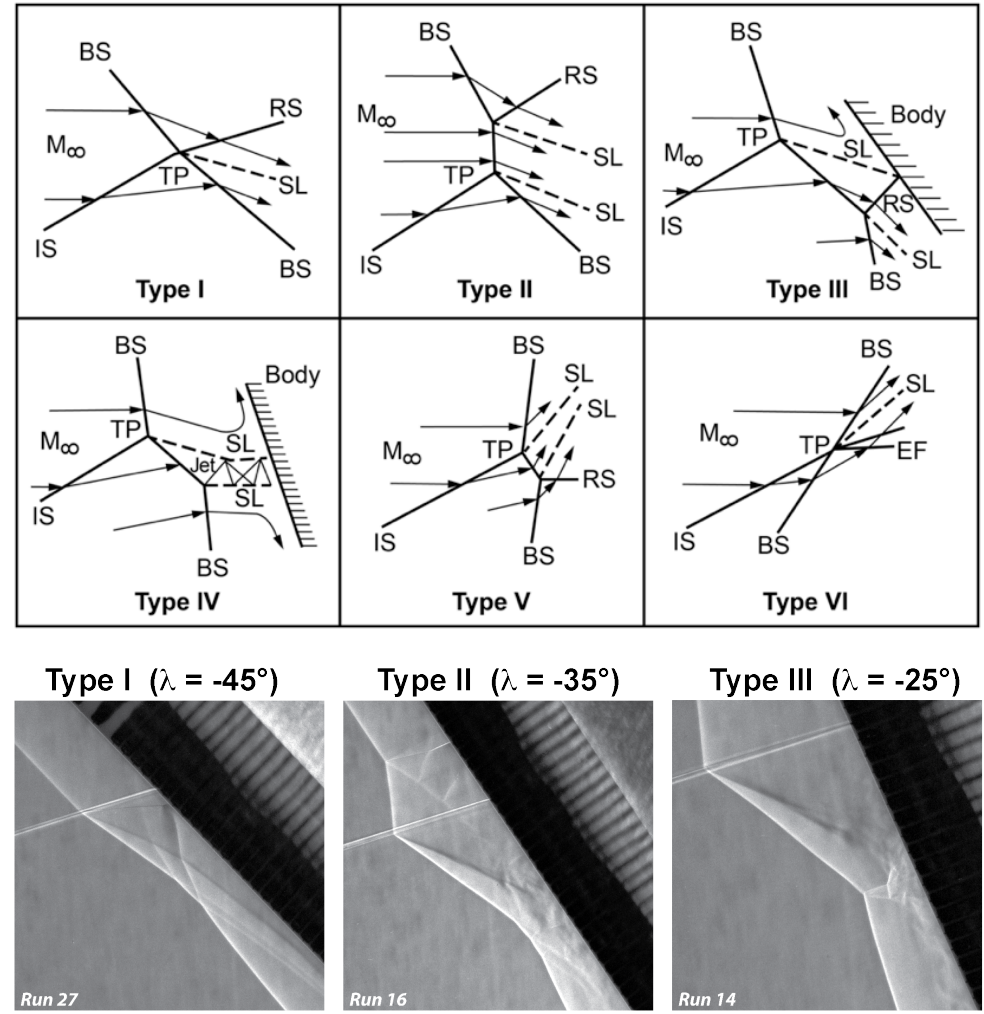

Type II $\left(\lambda=-35^{\circ}\right)$
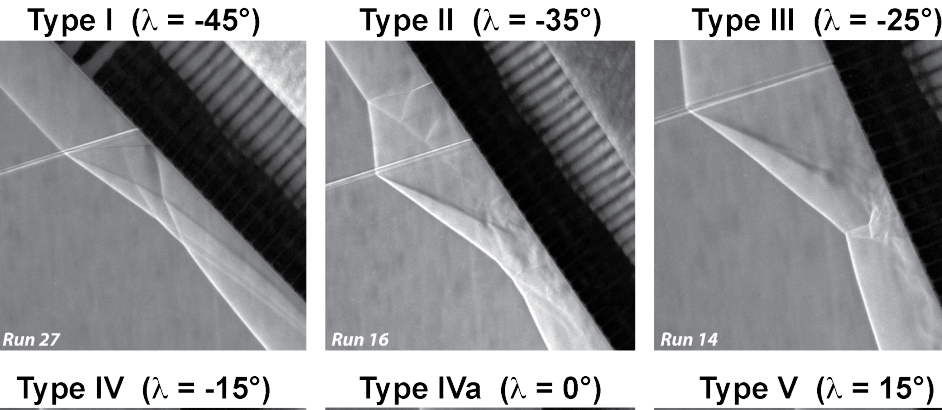

b)

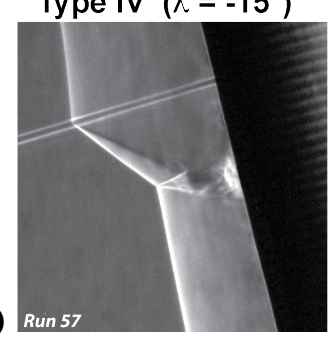

Type IVa $\left(\lambda=0^{\circ}\right)$
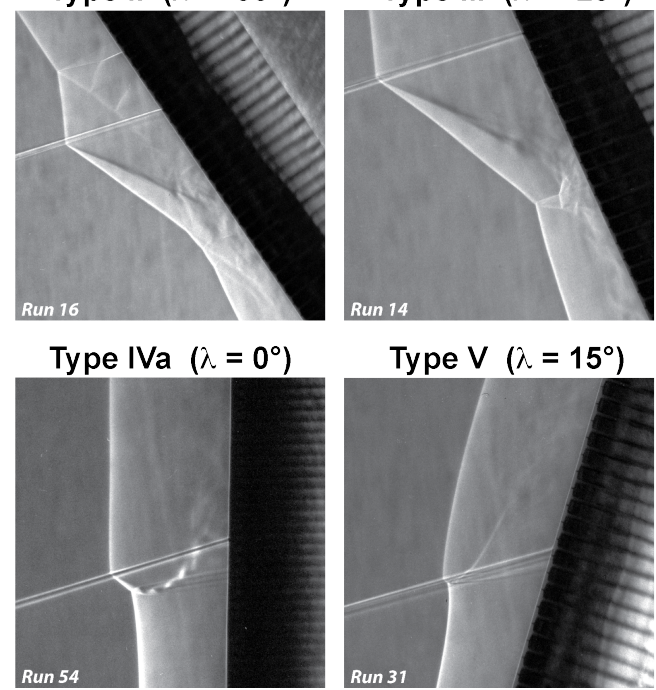

Type $V\left(\lambda=15^{\circ}\right)$

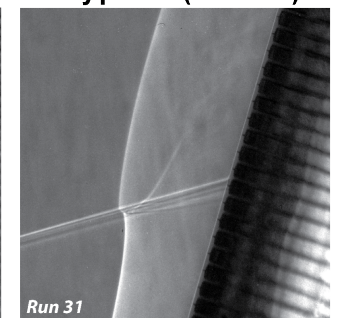

Figure 1. a) Edney ${ }^{1}$ catalogued six types of shock interactions (IS $=$ incident shock, $B S=$ bow shock, $R S=$ reflected shock, $\mathrm{EF}=$ expansion fan, $\mathrm{TP}=$ triple point, $\mathrm{SL}=$ shear layer, $\mathrm{M}_{\infty}=$ free-stream Mach number). b) Photographic examples of these shock types (including Type IVa, but excluding Type VI) from Test 6692. ${ }^{11}$

The results of the current study contribute to the knowledge of 3D shock-on-strut interactions in hypersonic flight. This research is the first known published study in which global thermal imaging techniques are used in conjunction with multi-dimensional thermal analyses to investigate high heating rates associated with shock-shock interactions. The phosphor thermography technique provided temperature and heat transfer data with an increased spatial resolution compared to the discrete sensors typically used in prior shock-shock interaction studies. Additionally, the current study provides information about shock-shock interactions from improved experimental tools such as high-speed zoom schlieren and two separate oil-flow techniques. 


\section{Facility}

The facility used for the present study was the 20-Inch Mach 6 Air Tunnel in the Langley Aerothermodynamics Laboratory at the NASA Langley Research Center (LaRC). ${ }^{12}$ This perfect gas facility has well-characterized flow uniformity and composition. ${ }^{13}$ The tunnel reservoir stagnation pressure and temperature, $P_{\mathrm{t}, 1}$ and $T_{\mathrm{t}, 1}$, are accurate to within $\pm 2 \%$. The LaRC 20-Inch Mach 6 Air Tunnel is a blow-down wind tunnel. Dry air from two high-pressure bottle fields is transferred to a 600-psia reservoir, where an electrical resistance heater heats the air to a maximum temperature of $1000^{\circ} \mathrm{R}$. The flow passes through two filters rated for $10-\mu \mathrm{m}\left(3.94 \times 10^{-4}\right.$-in. $)$ and $5-\mu \mathrm{m}\left(1.97 \times 10^{-4}\right.$-in. $)$ particles, respectively, that are installed between the heater and the settling chamber before entering the 20.5 in. by 20 in. test section at Mach 6. The top and bottom walls of the two-dimensional nozzle are contoured and the side walls are parallel. This wind tunnel exhausts either into combined $41-\mathrm{ft}$ diameter and $60-\mathrm{ft}$ diameter vacuum spheres, a 100 -ftdiameter vacuum sphere, or to the atmosphere through an annular steam ejector. Free-stream flows with Mach numbers between 5.8 and 6.1 and unit $\mathrm{Re}_{\infty}$ numbers between $0.5 \times 10^{6} / \mathrm{ft}$ and $8.3 \times 10^{6} / \mathrm{ft}$ are possible in this facility. ${ }^{14}$

Models are typically mounted on the arc-sector injection system located in a housing below the test section. This injection system is used to quickly insert the model into the flow after the tunnel has been started. Aeroheating tests generally have total run times of $30 \mathrm{sec}$, with typical model injection times of approximately $1.5 \mathrm{sec}$ and a model residence time on the tunnel centerline of approximately 5-10 sec. The actual mean flow conditions for this study are provided in Table 1. These flow conditions were calculated by averaging the parameters for thirty runs at unit $\operatorname{Re}_{\infty}=$ $2.1 \times 10^{6} / \mathrm{ft}$ and two runs at unit $\operatorname{Re}_{\infty}=1.1 \times 10^{6} / \mathrm{ft}$. The parameters for a unit $\operatorname{Re}_{\infty}=4.1 \times 10^{6} / \mathrm{ft}$ correspond to the one run that was conducted at that unit $\operatorname{Re}_{\infty}$ number.

Table 1. Actual mean flow conditions in Tests 6976 and 6983 in 20-Inch Mach 6 Air Tunnel.

\begin{tabular}{|c|c|c|c|c|c|c|}
\hline $\mathrm{M}_{\infty}$ & $\mathrm{Re}_{\infty}, \mathrm{ft}^{-1} \times 10^{6}$ & $P_{\mathrm{t}, 1}, \mathrm{psia}$ & $T_{\mathrm{t}, 1},{ }^{\circ} \mathrm{R}$ & $\rho_{\infty}, \mathrm{x} 10^{-4} \mathrm{slug} / \mathrm{ft}^{3}$ & $T_{\infty},{ }^{\circ} \mathrm{R}$ & $U_{\infty}, \mathrm{ft} / \mathrm{s}$ \\
\hline 5.90 & 1.1 & 60.5 & 875.1 & 0.33 & 110.3 & 3035 \\
\hline 5.96 & 2.1 & 125.5 & 898.5 & 0.63 & 111.3 & 3082 \\
\hline 6.00 & 4.1 & 252.2 & 901.6 & 1.23 & 110.5 & 3087 \\
\hline
\end{tabular}

\section{Experimental set up}

Figure 2 shows a graphical representation of the test hardware utilized for this study. The key components in Fig. 2 are the SG, a flat plate with a sharp leading edge that produces a $16.7^{\circ}$ planar incident shock at Mach 6 , and the model, inclined at $15^{\circ}$ in the figure. Similar images of these computer-aided design models were used prior to the experiment to ensure the visibility of the leading edge through the wind tunnel windows for each configuration. In each case, the upper tip of the model was swept $0^{\circ}, 15^{\circ}$, or $25^{\circ}$ forward of vertical, defined as $0^{\circ},-15^{\circ}$, and $-25^{\circ}$ model angles of attack (AoA), respectively. Image a) in Fig. 2 also shows the arc sector positioned below the test section in the 20-Inch Mach 6 Air Tunnel. Ten bolts (five on each side) secure an I-beam to the arc sector using a 1in. thick spacer to center the model in the core flow of the wind tunnel. Three bolts on either side secure the strut to the I-beam. The strut head plate is bolted to the top surface of the strut to support the stainless steel SG (6 in. wide by $17 \mathrm{in.} \mathrm{long)} \mathrm{angled} 9^{\circ}$ from horizontal. A stainless steel support that allows height adjustments is bolted into the strut head plate behind the SG. This support holds the model above the SG using a 0.5 -in. diameter sting. The model is bolted to the sting through a component used to change the AoA of the model, called the "fin AoA adjuster".

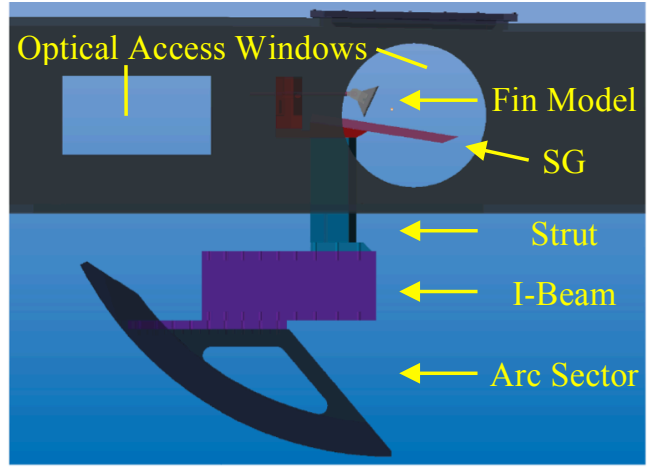

a)

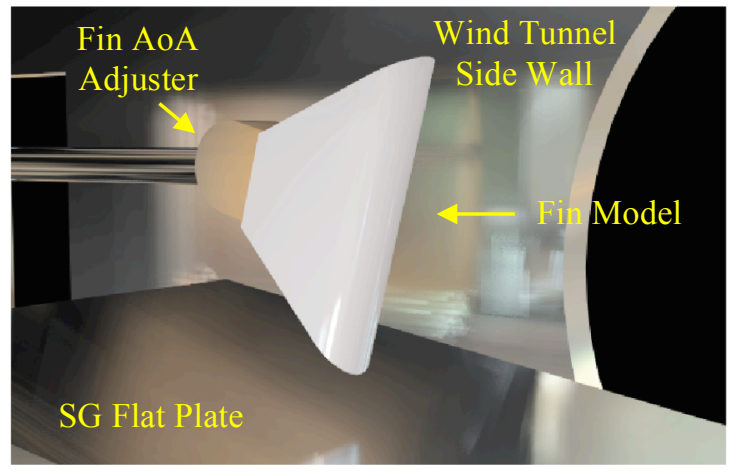

b)

Figure 2. Side-view renderings of the tunnel test section with a) the arc sector, I-beam, strut and other hardware, and b) the 0.5-in. radius fused silica model at $\mathbf{a}-15^{\circ} \mathrm{AoA}$ above the shock generator. 
In Fig. 3, the model support system is retracted into the "box" below the tunnel test section. A single thermocouple is embedded at the surface of each fused silica model just below the phosphor coating. The bead location for the 0.5 -in. radius model is labeled in the figure. These thermocouples measured the change in temperatures between the time the pre-run phosphor thermography image was recorded and the time that the run began to determine the proper offset for the leading-edge, pre-run temperatures input to the 1D and 2D finitevolume (FV) codes in the heat transfer analyses. The stainless steel fin AoA adjuster is shown more clearly in the inset image. A hinge pin with a retaining ring allows the model to be rotated through any AoA between $-45^{\circ}$ and $45^{\circ}$ from horizontal. An inclinometer was used to set the AoA of the fin. The model was always positioned between 0.5 and $0.75 \mathrm{in}$. above the flat plate SG or the strut head plate to eliminate interactions between the tip of the model and the flat plate boundary layer (BL) flow. Flow density gradients behind the models and shock-BL interactions with the flow over the SG are discussed more in Ref. 15.

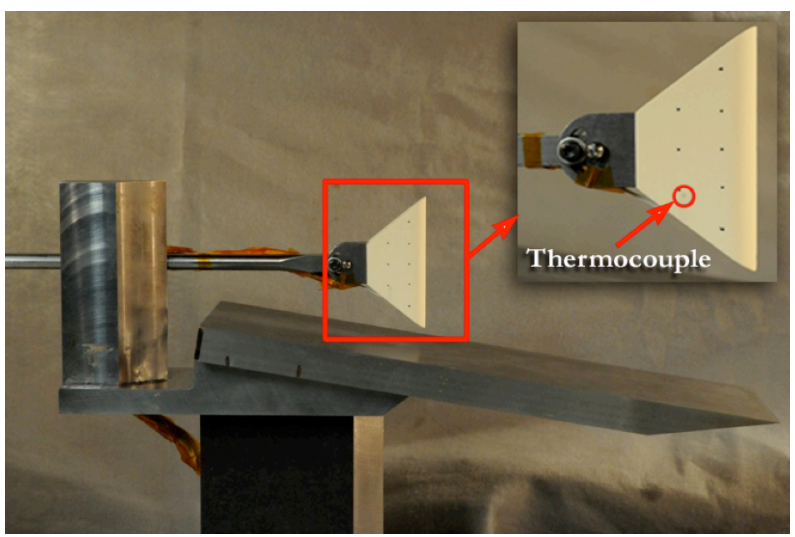

Figure 3. Fused silica (0.50-in. radius) model with a thermocouple near the bottom and a close-up inset image showing the thermocouple bead.

Oil-flow and schlieren data were collected to visualize surface streamlines and flow density gradients in the vicinity of the off-surface shock-shock interactions to provide insight into the flow behavior. The surfaces of 15-5 stainless steel models, cut from a wire electrical discharge machine, were polished smooth and covered with multiple layers of Rust-Oleum ${ }^{\circledR}$ high-temperature BBQ flat black paint (heat resistant up to $1200^{\circ} \mathrm{F}$ ). This coating provided sufficient contrast with a white-pigmented oil in videos and images during and after the oil-flow wind tunnel runs. Two oil-flow techniques were used. In the first method, a clear base coat of 350 centipoise (cP) oil was applied to the model and dots of $350 \mathrm{cP}$ oil mixed with a titanium white pigment were flicked onto the model surface that merged into streaks of oil along the flow streamlines. For the second method, the model was uniformly covered with a full layer of $350 \mathrm{cP}$ oil mixed with pigment to reveal regions of high or low shear on the model. The flow density gradients were recorded with a custom zoom schlieren system that used a set of lenses with a continuous light source to magnify a smaller region of interest encapsulating the shock-shock interaction in the flow. More details about the oil flow and schlieren techniques are provided in Ref. 15.

The mostly solid, porous fused silica models facilitated a 2D conduction assumption through a half cylinder to represent the model leading edge in the $\mathrm{FV}$ heat transfer codes. These models were covered with a phosphor mixture composed of $\mathrm{ZnCdS}: \mathrm{Ag}$, Ni and $\mathrm{La}_{2} \mathrm{O}_{2} \mathrm{~S}$ : $\mathrm{Eu}^{3+}$. These components work together as a two-color relative intensity phosphor coating that is sensitive to temperature changes on the surface of a wind tunnel model. Fiducial marks are dots applied to a model in specified locations that are used to correlate distances between features in an image to the physical dimensions of the model. Fiducial marks were applied to the oil-flow models (using orange Testors ${ }^{\circledR}$ fluorescent enamel paint) and the phosphor-coated models (using Dykem ${ }^{\circledR}$ Hi-Spot blue ink ${ }^{16}$ ) in the same pattern for all the models that share the same nose radius. The model fabrication process is further discussed in Ref. 15 .

Phosphor thermography was implemented to obtain a temporal record of surface temperatures for the models during the wind tunnel runs. Merski discusses a data reduction program called Imaging for Hypersonic Experimental Aeroheating Testing (IHEAT) used to obtain quantitative wind tunnel aeroheating data. ${ }^{17,18}$ This phosphor thermography method routinely is used to determine the global surface heating distribution on hypersonic wind tunnel models. In the baseline heating cases without a shock-shock interaction, the phosphor thermography images were recorded at $10 \mathrm{fps}$ after the initial images were taken when the model reached the wind tunnel centerline. During the runs with a shock-shock interaction, the phosphor data was acquired at $30 \mathrm{fps}$ to maximize the amount of data obtained before the leading-edge temperatures exceeded the phosphor system limit. The thermocouple data was obtained at $30 \mathrm{~Hz}$. 
Figure 4 shows the marked models in images a) to c), including the backup (left side) and primary (middle) fused silica models, and the metal oil-flow models (right side). The images in Fig. 4d) and e) show the 0.75-in. radius metal model (top image), without a coating of oil, and the 0.50 -in. radius fused silica model (bottom image) in the tunnel test section. These models are illuminated with ultra-violet light to reveal the fluorescent fiducial marks and the phosphor coating, respectively. The 20-Inch Mach 6 Air Tunnel runs were conducted either without an SG installed or with a $9^{\circ} \mathrm{SG}$ angle. For most of these runs, the nominal Mach number was 5.96 and the unit $\mathrm{Re}_{\infty}$ number was $2.1 \times 10^{6} / \mathrm{ft}$. A unit $\mathrm{Re}_{\infty}$ number sweep was conducted with two runs at $1.1 \times 10^{6} / \mathrm{ft}$, one run at $2.1 \times 10^{6} / \mathrm{ft}$, and one run at $4.1 \times 10^{6} / \mathrm{ft}$ for the 0.25 -in. radius model at a $-15^{\circ}$ AoA. The model AoA was either $0^{\circ},-15^{\circ}$ or $-25^{\circ}$ in each case to yield a Type IVa, a Type IV or a Type III interaction. These shock-shock interaction types were visually confirmed using high-speed schlieren data obtained with a Phantom 9 (1600fps) and a Phantom 12 (7900 fps) camera. Phosphor thermography data was first obtained with the entire fin leading edge in the field of view (referred to as zoomed out) and in a separate run with the camera focused on the region of interest (or zoomed in). The second run was performed to maximize the spatial resolution of the acquired data in the shock-shock interaction region. Additional information about the data acquisition processes and the run matrix is provided in Ref. 15.

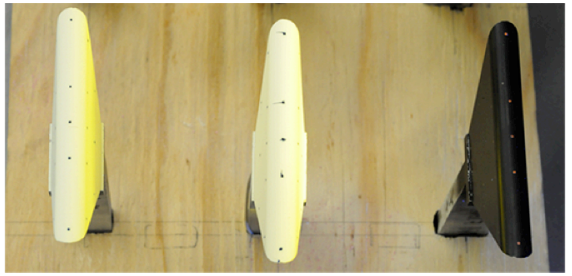

a) $r_{\text {nose }}=0.25$ inches

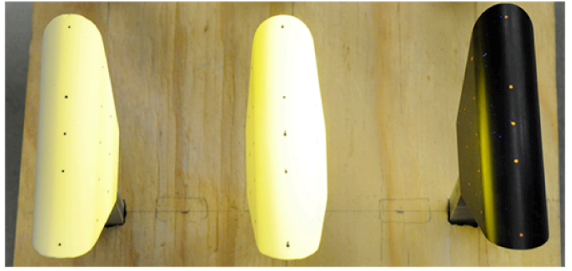

b) $r_{\text {nose }}=0.50$ inches

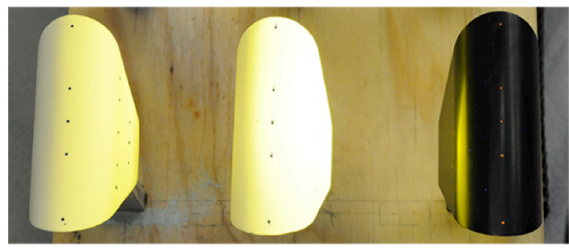

c) $\mathbf{r}_{\text {nose }}=0.75$ inches

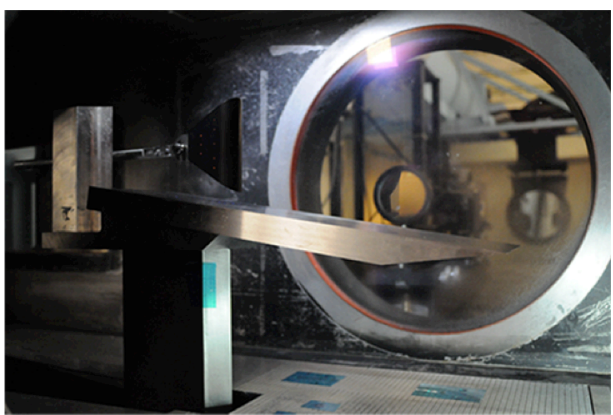

d)

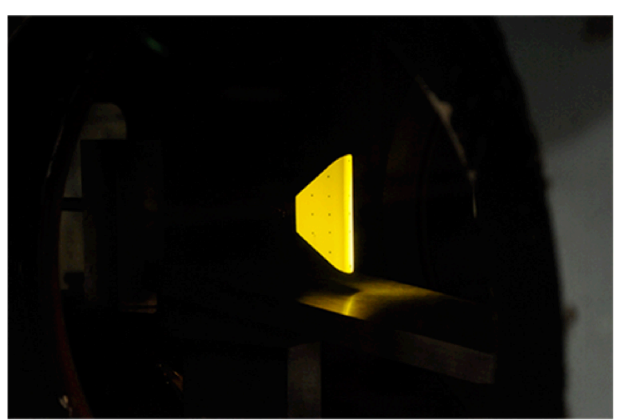

e)

Figure 4. The left images a) to c) show the primary and backup fused silica models and the metal oil flow models. The right images show d) a metal model (0.75-in. radius) and e) a fused silica model (0.50-in. radius) inserted in the tunnel with ultra-violet illumination.

\section{Heat transfer analysis}

Surface heat transfer coefficients based on the temperature readings for the fused silica models were calculated using IHEAT and 1D and 2D FV conduction codes. These programs were used to determine the optimum method to predict heating profiles if models are exposed to sharp temperature gradients, which in this study were produced by shock-shock interactions. The IHEAT code is the primary method used to analyze phosphor thermography data obtained in Langley's hypersonic facilities. IHEAT is a 1D code that assumes the model is semi-infinite in the through-thickness dimension, so heat applied at the surface does not reach the back of the model during a short wind tunnel run. Convective heat transfer coefficients, $c_{h}$, are calculated from a convective heat transfer, $\dot{q}_{c o n v}$, equation based on an enthalpy difference, i.e.,

$$
\dot{q}_{c o n v}=c_{h}\left(h_{a w}-h_{w}\right)
$$

Additional assumptions and equations incorporated in the IHEAT code are described in Ref. 17 and Ref. 18. 
Two direct, finite-volume codes were written in Fortran to approximate the conduction in the phosphor-coated fused silica models. The 1D code assumed heat was conducted only through the thickness of the model, while the 2D code also considered conduction in the lateral direction. The purpose of the 1D FV code in the present study is to provide a direct comparison between the 1D algorithm in IHEAT and the assumptions in the FV codes for the 1D versus $2 \mathrm{D}$ comparison. Both the IHEAT and $1 \mathrm{D}$ FV codes neglect the effect of surface curvature.

The diagram in Fig. 5 shows the cylindrical geometry used in the 2D code to approximate the model leading edge. To simplify the calculations, the code assumes a cylinder with dimensions equal to the leading-edge length of $4 \mathrm{in}$. and the appropriate nose radius. A rectangle bounded by the stagnation line on the leading edge and the centerline of the cylinder (in red in Fig. 5) defines the boundaries of the nodes in both FV codes. The 2D code used cylindrical cell volumes that surrounded nodes in the rectangular plane. Definitions of the areas and volumes around the nodes in the 2D grid, as well as the number of volumes and the corresponding spacing in the radial and lateral directions, are provided in Ref. 15. The coordinate systems in the figure apply to both FV codes.

Two boundary conditions are applied to the grid of finite volumes in the 1D code, and four boundary conditions are applied in the 2D code. A Dirichlet boundary condition is applied at the surface $(r=R)$ in both codes using the known temperatures, $T_{d}$, of the models at each time step. An adiabatic boundary condition (no heat transfer) is assumed at the cylinder centerline $(r=0)$ in both codes. The boundary conditions at the top $(z=L)$ and at the bottom $(z=0)$ of the cylinder in the lateral direction are also adiabatic in the 2D code. Adiabatic boundaries are assumed because temperature data is not available for nodes outside of the rectangular plane. Thus, the discrete heat transfer expressions that include nodal information external to the boundary nodes are assumed to be equal to zero.

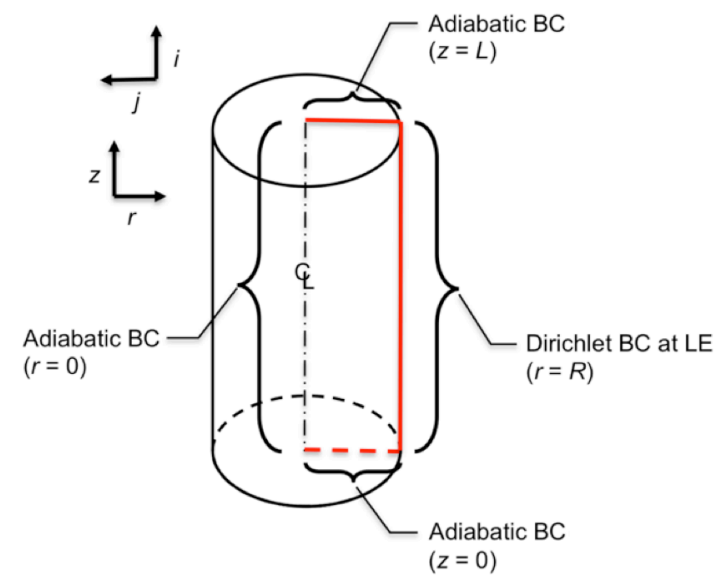

Figure 5. Overall view of the boundary conditions of the cylindrical leading edge modeled in the 1D (neglecting curvature) and 2D (using cylindrical coordinates) FV codes.

The 1D code uses an FV formulation of the Crank-Nicolson algorithm to solve the discretized conduction equation in the radial direction. The Crank-Nicolson method employs the trapezoidal rule of time integration in which the discrete spatial temperature gradient is evaluated as an average of the gradient at the current $(n)$ and future $(n+1)$ time steps. ${ }^{19}$ This method yields an unconditionally stable time-marching scheme in the 1D FV code. The resulting system of equations forms a tri-diagonal system matrix that is multiplied by the temperatures at the central nodes of each cell. These equations comprise a system matrix used in the implicit Thomas Algorithm ${ }^{20}$ to solve for the nodal temperatures.

The $2 \mathrm{D}$ conduction equation is discretized using an $\mathrm{FV}$, unconditionally stable, alternating direction implicit algorithm. ${ }^{20}$ This method sweeps through the geometry of the model twice for every time step, first in the " $z$ " direction (along the fin leading edge) for all the nodes and then in the " $r$ " direction (radially) using an updated temperature distribution, $T^{*}$, at the intermediate time step $(\Delta t / 2)$. Chapter 4 in Ref. 15 describes the equations used to calculate an initial temperature distribution in the 1D and 2D codes. The heat transfer coefficients are calculated at the end of each full time step in both the 1D and 2D FV codes. An energy balance between the stored heat and the radiation, convection, and $2 \mathrm{D}$ conduction heat transfer at the model surface is given by

$$
\begin{aligned}
\rho c_{P} V \frac{\partial T}{\partial t}= & \frac{\partial}{\partial r}\left(A_{\text {cond }} k \frac{\partial T}{\partial r}\right)+\frac{\partial}{\partial z}\left(A_{\text {cond }} k \frac{\partial T}{\partial z}\right)-\left[A_{\text {rad }} \varepsilon \sigma\left(T_{i, j}^{4}-T_{t w}^{4}\right)\right] \\
& -A_{\text {conv }} c_{h}\left(h_{a w}-h_{w}\right)
\end{aligned}
$$


The variables $A_{\text {cond }}, A_{\text {rad }}$ and $A_{\text {conv }}$ represent the areas through which heat is transferred by conduction, radiation, and convection, respectively. These variables vary depending on the volume $V$ of the cell for which the heat transfer is computed. The variable $\varepsilon$ is the emissivity, $\sigma$ is the Stefan-Boltzmann constant, $T$ is the temperature at a given location (either at each node specified by $i$ in the $1 \mathrm{D}$ code or $i, j$ in the $2 \mathrm{D}$ code, or at the tunnel wall, $t w$ ), $h_{a w}$ is the adiabatic wall enthalpy and $h_{w}$ is the enthalpy of the air at the surface temperature of the model. The density, $\rho$, specific heat, $c_{p}$, and thermal conductivity, $k$, in the heat transfer equation are shown as constant values. In both the $1 \mathrm{D}$ and 2D FV conduction codes, the thermal properties of $c_{p}$ and $k$ depend on temperature and are updated after each full time step. The left side of equation (2) represents energy stored in the object due to a temporal temperature gradient, $\partial T / \partial t$. The right side represents heat conducted through the object due to a second-order spatial temperature gradient, either $\partial^{2} T / \partial r^{2}$ or $\partial^{2} T / \partial z^{2}$. After the heat transfer equation is properly discretized, the equation is rearranged to solve for the enthalpy-based heat transfer coefficient, $c_{h}$, for each surface volume at a nondimensional position specified as the spatial location $x$ (which is equal to 0 at the lower tip) divided by the full length $L$ (4 in.) of the leading edge, as shown in Fig. 6a) for a 0.25 -in. radius model at a $0^{\circ}$ AoA.

The FV codes output a dimensional heat transfer coefficient based on the temperature data at each pixel along the leading edge of the model. The $1 \mathrm{D}$ code loops through input data to derive a heat transfer coefficient at every pixel individually, while the $2 \mathrm{D}$ code calculates the data for the full leading edge using a line implicit scheme. These heat transfer coefficients are then non-dimensionalized to determine the relative magnitude of the peak heating in the shock-interaction region compared to the baseline heating (no interaction). The heat transfer coefficients at $x / L=$ 0.75 to $x / L=0.85$ along the leading edge (relative to the $x / L$ locations in Fig. 6) are relatively far away from both the peak heating region and the leading-edge fiducial marks. In the runs for which the full leading edge was visible in the image, these coefficients were averaged to provide a reference coefficient to derive the non-dimensional data.

For some of the wind tunnel runs in which the camera is zoomed in on the shock interaction region, baseline heating data is not available. To be consistent, each zoomed-in run used the average value from the corresponding zoomed-out run as a reference value to calculate non-dimensional data. The model surface temperatures vary slightly between runs, so this assumption yields lower non-dimensional peak heat transfer coefficients in some of the zoomed-in cases, as evidenced by the cases in which data outside the shock interaction region is available and is less than a non-dimensional baseline value of 1.0. This assumption is necessary to compare the non-dimensional data for higher (zoomed-in) and lower (zoomed-out) spatial resolutions.

A comparison in Fig. 6b) demonstrates the potential error associated with reducing the zoomed-in leading-edge data by a reference heat transfer coefficient from a separate wind tunnel run. If an average of the zoomed-in heat transfer data from Run 44 (between $x / L=0.38$ and $x / L=0.41$ ) is used to obtain non-dimensional heat transfer coefficients, the peak heat transfer coefficient $c_{h} / c_{h, r e f}$ is 4.3 . However, if the reference value from Run 43 (averaged over $x / L=0.75$ to $x / L=0.85$ ) is used, the peak $c_{h} / c_{h, \text { ref }}$ is only 3.5 . If data in Run 44 in the region between $x / L=0.75$ and $x / L=0.85$ were available to be used for a reference, non-dimensional data from this run likely would lie somewhere between the two curves in the plot, since the average heat transfer in the region from $x / L=0.38$ to $x / L=$ 0.41 is typically slightly lower (i.e., by a difference of 0.015 for Run 43 ) than in the $x / L=0.75$ to $x / L=0.85$ region.

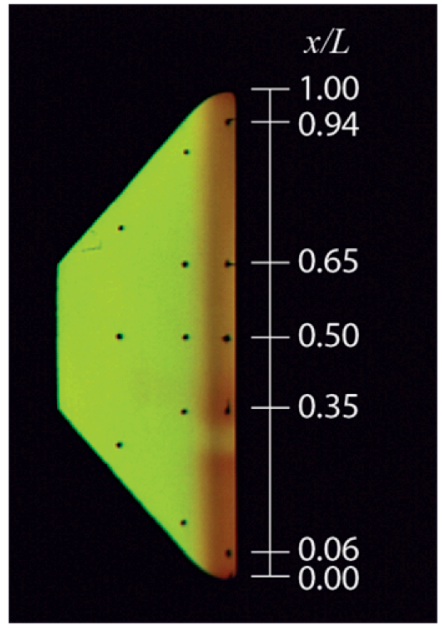

a)

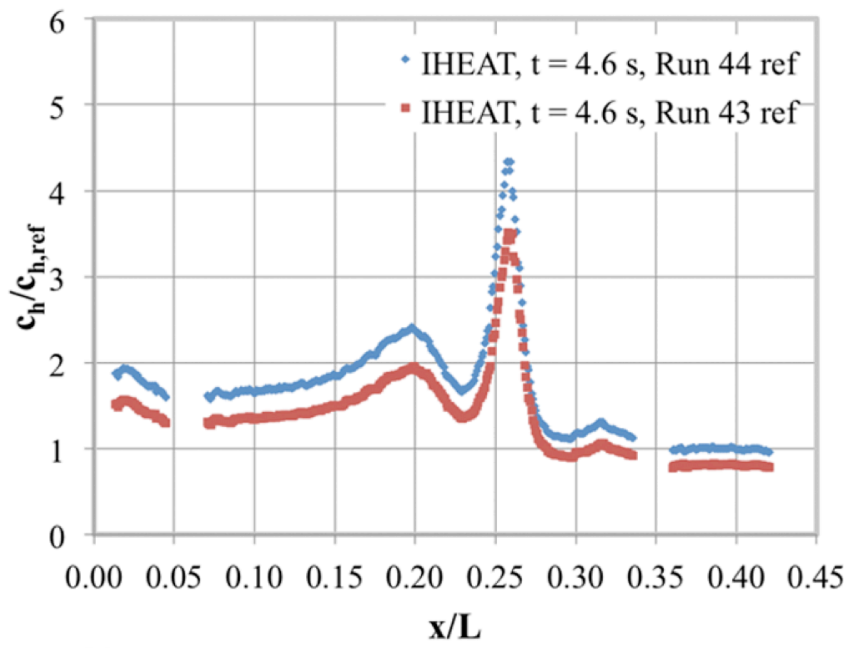

b)

Figure 6. a) Diagram of $x / L$ locations for the 0.25 -in. radius model. b) Heat transfer coefficients for the zoomed-in case for the same model at $\mathrm{a}-15^{\circ} \mathrm{AoA}$ and a unit $\operatorname{Re}_{\infty}=1.1 \times 10^{6} / \mathrm{ft}$ (using two reference values). 


\section{Experimental measurements and results}

The effect of fin sweep angle and leading-edge radius on the shock interaction patterns and resulting surface heating are presented by comparison of zoom schlieren, oil-flow and heat transfer results. The specific fin sweep angles examined in the present study are $0^{\circ},-15^{\circ}$, and $-25^{\circ}$, where the negative angle denotes the fin is swept forward from the base. The latter two sweep angles provide cases with strong lateral temperature gradients due to the Type IV and Type III shock-shock interactions, respectively, and the $0^{\circ}$ sweep angle yields a lower heating case for a Type IVa interaction with smaller lateral temperature gradients. These cases were selected to assess the need for a two-dimensional analysis method. Also, three model geometries were tested to determine how the features of each type of interaction change due to the leading-edge radius. The following discussion is a preface to the subsequent results and analysis.

In the 2D schlieren images, the planar incident shock is seen as a line. The incident shock does not impinge on the model but instead wraps around the bow shock. The "extrapolated incident shock location" described in the following images references the location where the incident shock would impinge on the leading edge in the absence of the bow shock. In computed schlieren images of the stagnation plane derived from computational results (not included here), the incident shock does not appear to continue through the bow shock. ${ }^{15}$ Experimental zoom schlieren data initially was obtained at a framing rate of $1600 \mathrm{fps}$. Additional data obtained at $7900 \mathrm{fps}$ was insufficient to resolve the unsteadiness observed in the $1600 \mathrm{fps}$ data, so the data acquired at $1600 \mathrm{fps}$ is presented.

The Type III and IV interactions provided peak heat transfers that rapidly exceeded the maximum phosphor thermography temperature limit. For that reason, only temperature images acquired about $0.2 \mathrm{~s}$ after the model reached the wind tunnel centerline (or at a time $t=1.8 \mathrm{~s}$ after the model injection sequence started) were used in the heat transfer analysis. In most cases, all the temperatures in these early line cuts were within the phosphor limits, permitting a comparison of the trends in the heat transfer coefficients due to each shock-shock interaction for the three leading-edge radii. A temporal collapse of the leading-edge line cuts and an analysis of the trends in heat transfer coefficients in specific surface locations over time were used to determine when to take the line cuts. ${ }^{15}$

The $\mathrm{x}$-axis in the heat transfer plots shows the non-dimensional $x / L$ locations for the 0.25 -in. radius model (referred to as $x / L_{0.25}$ ). These locations are offset for the 0.50 - and 0.75 -in. radius models to align the notable features of the line cuts (either the valley for the Type IVa interaction or the peaks for the Type III and IV interactions). Gaps in the line cuts along the leading edge indicate the removal of fiducial mark data. Five fiducial mark locations were used to align the model vertically in the wind tunnel and to guarantee that at least two fiducial marks are visible when the camera is zoomed in on the interaction region.

Contour maps of the 1D Fay-Riddel1 ${ }^{21}$ non-dimensionalized heat transfer coefficients from IHEAT are presented at $t=1.8 \mathrm{~s}$. The limits on the color bar scale in each contour map were set to zero and three to ensure the main features of the heat transfer pattern are visible for every test configuration. The average baseline heating data in the region away from the shock-shock interaction during the wind tunnel run were used to determine the relative heating augmentation in the presence of an interaction for the heat transfer line cuts. Since different reference values were used to convert the heat transfer contour maps and the line cuts to non-dimensional data, the y-axis scale in the plots does not directly correlate to the color bar on the contour maps.

Uncertainties in the phosphor thermography data depend on the rise in the model surface temperatures. The following values of uncertainty are based on historical tests with a variety of types of models. On surfaces with a significant temperature rise $\left(>70^{\circ} \mathrm{F}\right)$, uncertainties are in the range of $\pm 10 \%$. For moderate temperature increases $(20$ $30^{\circ} \mathrm{F}$ ), the uncertainties are roughly $\pm 25 \%$. More information on phosphor thermography uncertainties is found in Ref. 17 and Ref. 18. Error bars are not included in the plots due to the density of the data in the line cuts.

The peak heat transfer coefficient in the shock-shock interaction region increases with increasing leading-edge radius in the non-dimensional line cuts. This trend is the opposite from the peak behavior in dimensional line cuts. This reversal in the leading-edge radius effects occurs because the reference value used to convert the heat transfer coefficients to non-dimensional values decreases as the leading-edge radius increases, which amplifies the peak heat transfer value for the larger model geometries. Non-dimensional heat transfer line cuts are presented to estimate the peak heating augmentation relative to the expected heat transfer in the absence of a shock-shock interaction.

Most of the following data were captured at a unit $\mathrm{Re}_{\infty}=2.1 \times 10^{6} / \mathrm{ft}$. However, for the FV comparisons near the end of this results section, data from runs with a unit $\mathrm{Re}_{\infty}=1.1 \times 10^{6} / \mathrm{ft}$ were used to ensure more time steps of useful heating data were available before the phosphor limit was exceeded in the shock-shock interaction region.

\section{A. Fin sweep of $0^{\circ}$}

Figure 7 displays schlieren images of the $0.25-, 0.50-$, and 0.75 -in. radius models, from left to right, at a $0^{\circ}$ AoA. The shock interaction is so close to the incident shock in Fig. 7a) that the Type IVa features are difficult to distinguish, though a close inspection reveals a very narrow supersonic jet with shear layers turned upward. The 
extrapolated incident shock is further separated from the supersonic jet and the curled-up shear layers for the 0.50in. radius model in Fig. 7b). The shear layers appear to attach to the surface of the model above the location where the incident shock wraps around the bow shock, which is similar to the behavior demonstrated in Fig. 1b).

The bow shock standoff distance is greatest for the 0.75-in. radius model, so the features of the Type IVa interaction are easier to identify for that model. A narrow supersonic jet extends nearly horizontally from the location where the bow shock is nearly vertical, before the jet turns upward and the shear layers that bound the jet spread apart to impinge on the surface of the model. A pattern of triangles that comprise the shock train formed by reflected shocks is visible near the beginning of the supersonic jet. The horizontal feature behind this shock train is likely a 2D projection of 3D flow density gradients that wrap around the model. The shear layers curve up past the extrapolated incident shock line, as was noted in videos of the schlieren data and is faintly visible in Fig. 7.

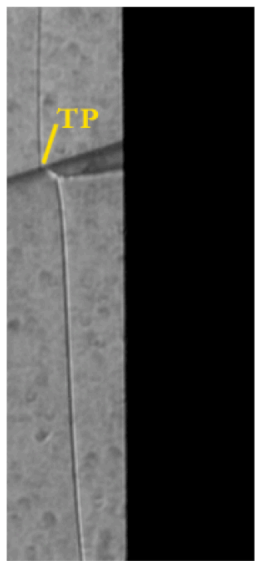

a)

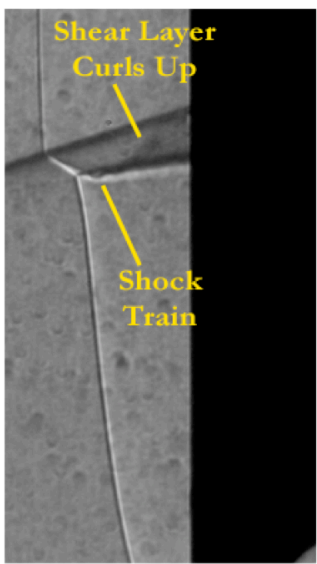

b)

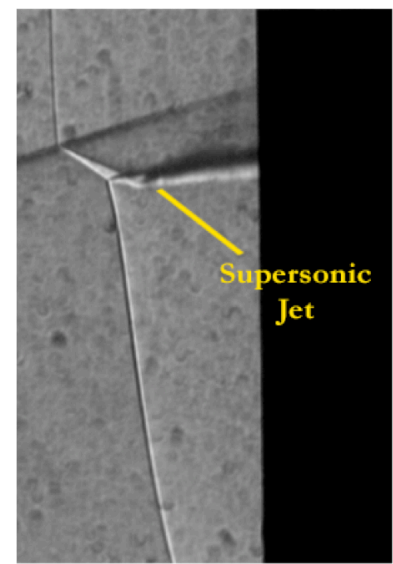

c)

Figure 7. Zoom schlieren images of a Type IVa interaction for a) 0.25-, b) 0.50- and c) 0.75-in. radius models at a $0^{\circ} \mathrm{AoA}$.

Oil flow images in Fig. 8 show characteristic streamlines and shear patterns for this Type IVa interaction with the 0.75 -in. radius model. The features in these oil-flow images are similar to the streamline patterns observed in the 0.25 - and 0.50 -in. radius models. Image b) indicates the oil movement for a model that was initially fully coated with oil, while the other images show streamlines on a model that was covered with dots of pigmented oil prior to the run. The oil-flow streamlines on the leading edge in Fig. 8 are fundamentally similar to the oil-flow streamlines in the region of the shock-shock interaction for a 0.5 -in. radius cylinder in Ref. 10.

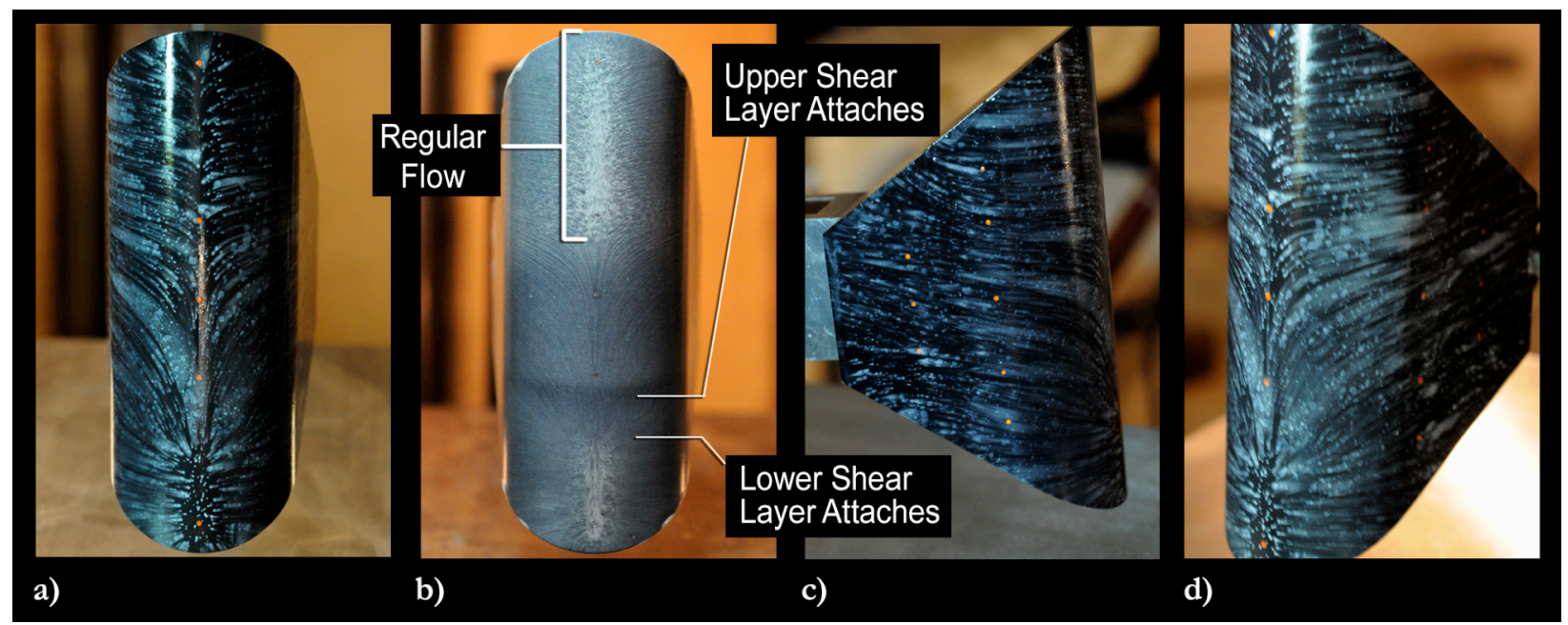

Figure 8. Oil-flow images of a Type IVa interaction for the 0.75-in. radius metal model at a $0^{\circ} \mathrm{AoA}$ for a) leading-edge view, dots, b) leading-edge view, full coating, c) right side view, dots, d) left side view, dots.

A horizontal line around the circumference of the leading edge in the full-coating image indicates the attachment point of the upper shear layer that curls up from the supersonic jet. Streamlines travel upward from this 
attachment point and then turn away from the leading edge. In the regions near the top and bottom of the model, away from the shock-shock interaction, the oil along the stagnation line did not move. The side oil flow patterns show nearly horizontal streamlines flow away from the leading edge and then turn toward the slanted edges of the model. The bow shock around the model at a $0^{\circ} \mathrm{AoA}$ is a nearly normal shock and, thus, the flow above the incident shock does not change direction but instead continues horizontally until the air reaches the surface of the model.

Edney ${ }^{1}$ states that a region of dead air exists along the leading edge below the shear layer attachment point in an oil-flow image of a 0.59 -in. radius cylinder exposed to a Type IVa interaction. Although this phenomenon is not clear in the oil flow images, the dead air region is visible in the IHEAT 1D contour maps in Figures 9 and 10.

IHEAT 1D contour maps of the heat transfer coefficients show zoomed-out (Fig. 9) and zoomed-in (Fig. 10) views of the $0.25-, 0.50-$, and 0.75 -in. radius models, from left to right, at a $0^{\circ} \mathrm{AoA}$. A blue gap between two green regions of higher heating along the model leading edge corresponds to a valley in the heat transfer coefficients in Fig. 11. This valley may be due to the "dead air" zone between the lower and upper shear layer attachment points on the model. This region shrinks, possibly due to conduction from the high heating areas, as the leading-edge radius increases. The green region of higher heating along the leading edge just below the dead air region on each model may be due to the flow travelling through both the incident and the bow shocks before reaching the surface of the model. The green heating region that spans the three middle fiducials in Fig. 9c) for the 0.75-in. radius model (which shrinks with decreasing model radius), is likely due to the curved shear layer attachment above the dead air region. The increased heating at the upper and lower tips of the model is due to edge effects at the boundary between the ceramic model and the surrounding air. A repeat run was conducted for the 0.50 -in. radius model at a $0^{\circ} \mathrm{AoA}$, and the heat transfer patterns were very similar between the two runs as expected. ${ }^{15}$

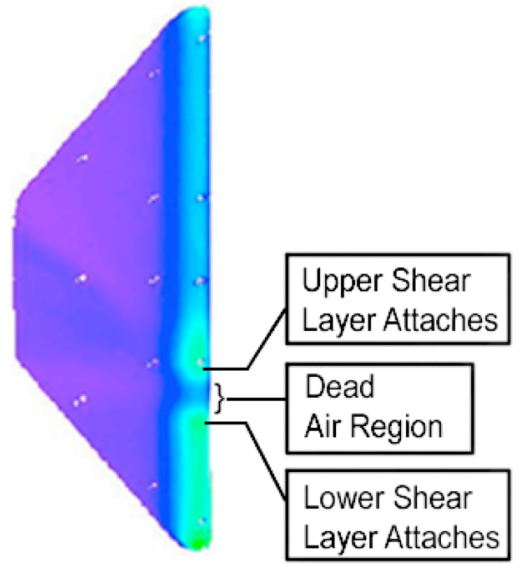

a)

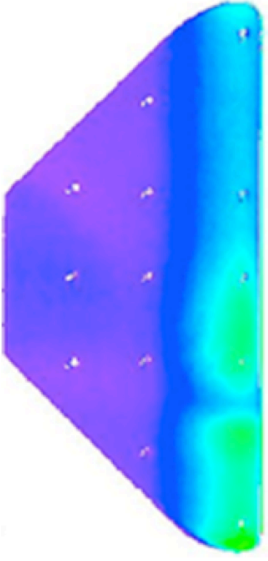

b)

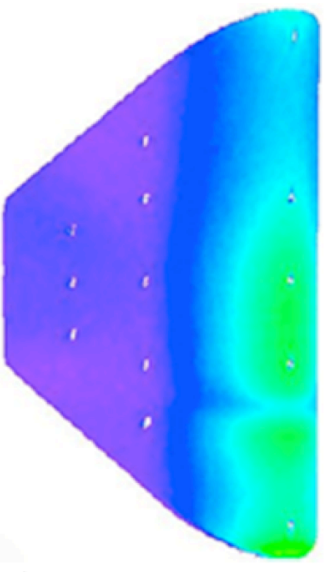

c)

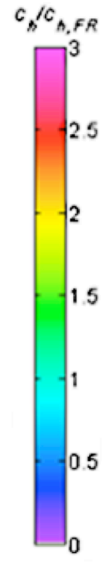

c) 0.75-in.

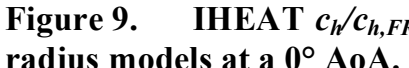

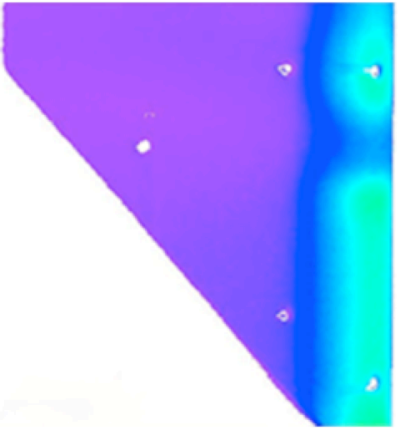

a)

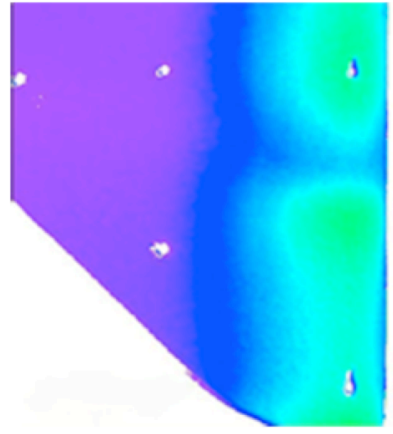

b)

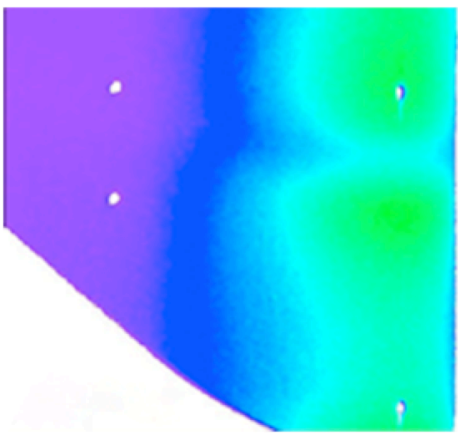

c)

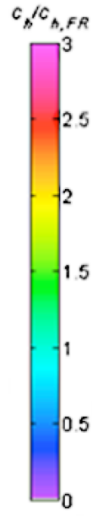

Figure 10. IHEAT $c_{h} / c_{h, F R}$ contour maps of a Type IVa interaction for a) 0.25-, b) 0.50-, and c) 0.75 -in. radius models at a $0^{\circ} \mathrm{AoA}$ (zoomed in). 
The Type IVa shock interaction yielded the lowest peak heat transfer augmentation of the interactions in this study. The line cut data for all three of the model geometries display similar features for the case with a $0^{\circ}$ AoA. Two small peaks of similar magnitude exist on either side of a trough in the heat transfer coefficients along the leading edge for this type of interaction. The non-dimensional heat transfer data along the leading edge for the three models is presented in Fig. 11. The first peak near $x / L_{0.25}=0.24$, with $c_{h} / c_{h, \text { ref }}$ approximately equal to 1.5 for the smallest model and about 1.8 for the larger models, corresponds to the lower shear layer attachment point on the leading edge near the extrapolated incident shock. Below that location on the leading edge, the heat transfer is relatively constant and elevated in each case relative to the baseline heating defined as $c_{h} / c_{h, \text { ref }} \approx 1$. The higher heat transfer coefficients from the lower tip of the model to the first peak are partially due to the flow passing through both the bow shock and the incident shock prior to contact with the surface of the model. The shear layer that forms the upper boundary of the supersonic jet attaches to the surface of the model to produce a second peak with a maximum magnitude between 1.4 for the 0.25 -in. radius model at $x / L_{0.25}=0.36$ and 1.8 for the 0.75 -in. radius model near $x / L_{0.25}=0.43$. The widths of the heat transfer peaks increase with increasing leading-edge radius, possibly due to conduction effects and the increased length of the shear layer prior to attachment. Edge effects yield increased heating at the upper and lower tips of the model for all three geometries in Fig. 9, which is clearly evident for the lower tip between $x / L_{0.25}=0$ and 0.1 in Fig. 11a). The line cut data for the two larger models were shifted to align features in the data since the model's position relative to the incident shock varied slightly with leading-edge radius.

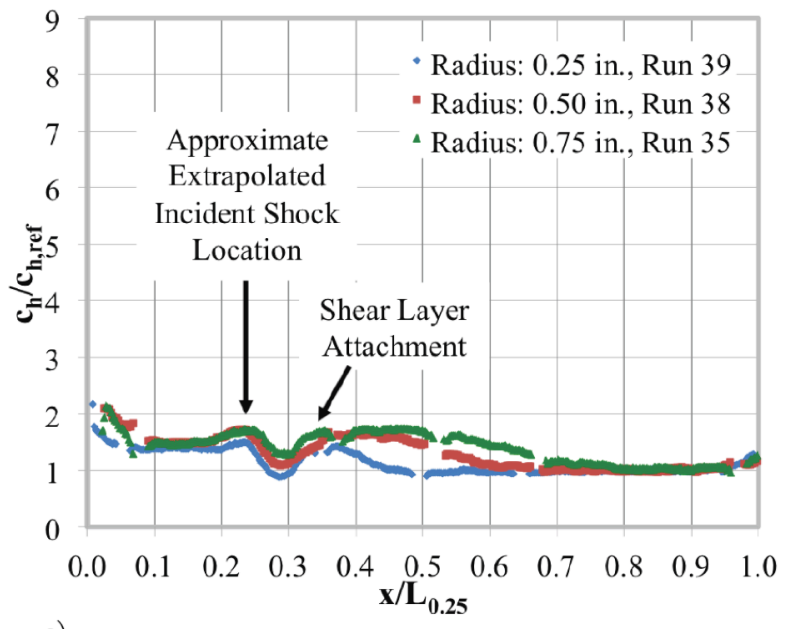

a)

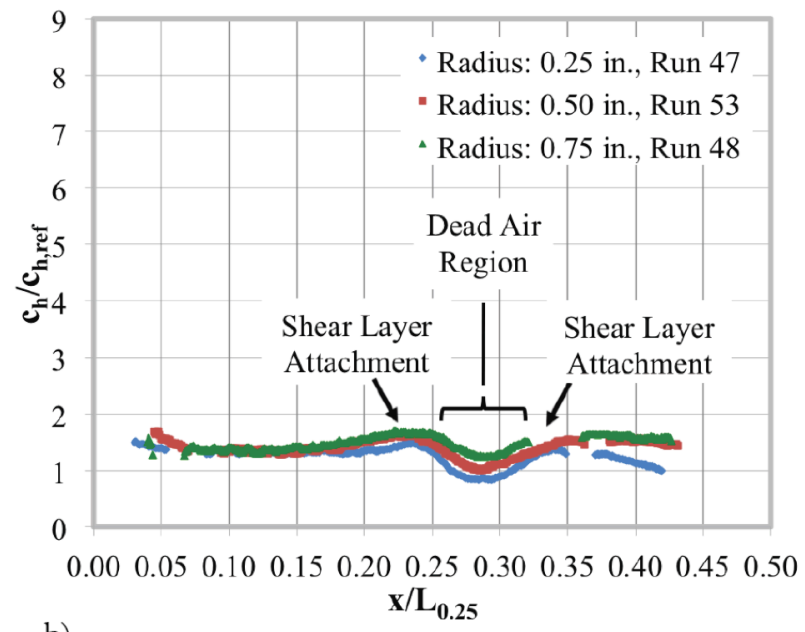

b)

Figure 11. Non-dimensional heat transfer coefficients at $t=1.8 \mathrm{~s}$ from the IHEAT code for a Type IVa interaction for the three fused silica models at a $0^{\circ} \mathrm{AoA}$ for a) zoomed-out and b) zoomed-in data.

\section{B. Fin sweep of $-15^{\circ}$}

The characteristic features of a Type IV interaction are visible in the schlieren images for the $0.25-, 0.50$ - and 0.75 -in. radius models (from left to right) at a $-15^{\circ}$ AoA in Fig. 12. One such feature is a supersonic jet emanating from the triple point that impinges nearly perpendicularly on the surface of the model. Schlieren videos of this interaction reveal changes over time in the density of the air between the bow shock and the model surface above the extrapolated incident shock location. The density gradient due to a vortex in that region is labeled in Fig. 12b) but is not very clear in the still images. Although the standoff distance differs, the shape of the bow shock above and below the interaction is similar for the models with varying nose radii.

Oil flow images for the 0.75 -in. radius model at a $-15^{\circ}$ AoA are provided in Fig. 13. These images display representative surface streamlines for a direct Type IV shock interaction. The shear associated with the supersonic jet impingement removes the majority of the oil in that region in the "full coating" images, labeled as b) and d) in the figure. The lower horizontal line in the two leading-edge images in this figure corresponds to the edge of this high shear region due to the supersonic jet. This line is also approximately located where the incident shock wraps around the model (outside of the bow shock) between the two lowest fiducials on the leading edge. The lower streamlines on either side of the stagnation line in Fig. 13a) resemble parabolas with a trough centered near the supersonic jet impingement. In the corresponding schlieren images, this supersonic jet impinges on the model in a narrow horizontal region, perhaps driving the parabolic streamlines away from the leading edge. 


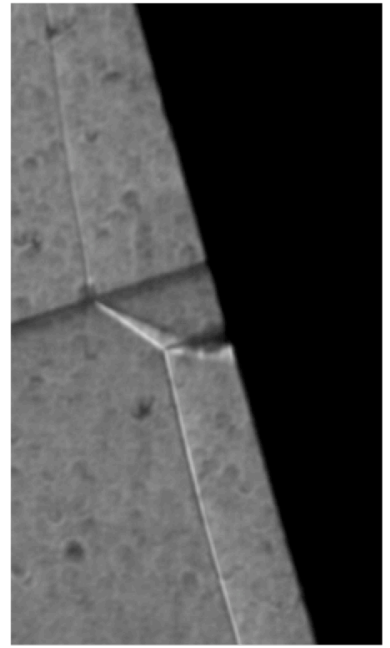

a)

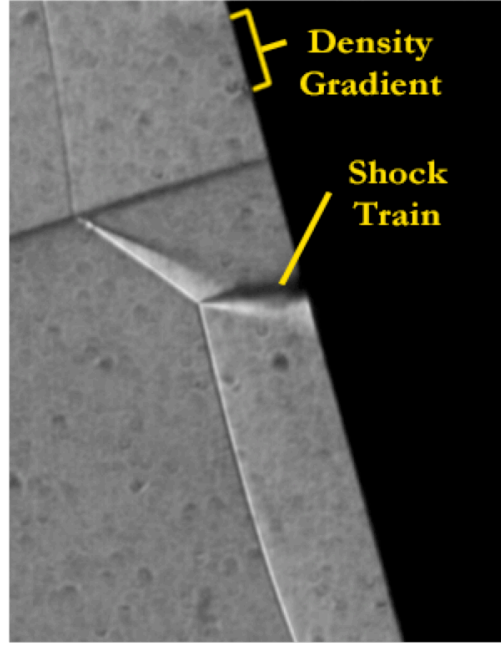

b)

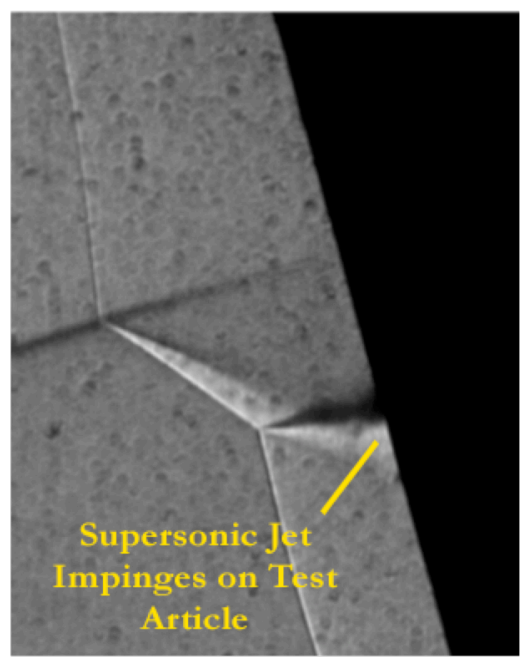

c)

Figure 12. Zoom schlieren images of a Type IV interaction for a) 0.25-, b) 0.50- and c) 0.75-in. radius models at a $-15^{\circ}$ AoA.

The upper horizontal line may be due to a vortex roll-up at the leading edge caused by two opposing flows meeting with the upper flow moving down and lower flow moving up. This unsteady vortex was observed as a density fluctuation in schlieren videos. A region of stationary dots in image a), or an undisturbed oil coating in image $b$ ), exists along the stagnation line near the top of the leading edge. The streamlines leading away from this region above the upper horizontal line indicate flow on an inclined cylinder at Mach 6. The c) and d) oil flow images in Fig. 13 reveal side views of the same model. A triangular shape in the middle on the side of the model indicates the vortex above the extrapolated incident shock also wraps around the surface of the model. The vortex in front of the leading edge likely continues along the sidewall and then splits up into this "v-shaped" or triangular region.

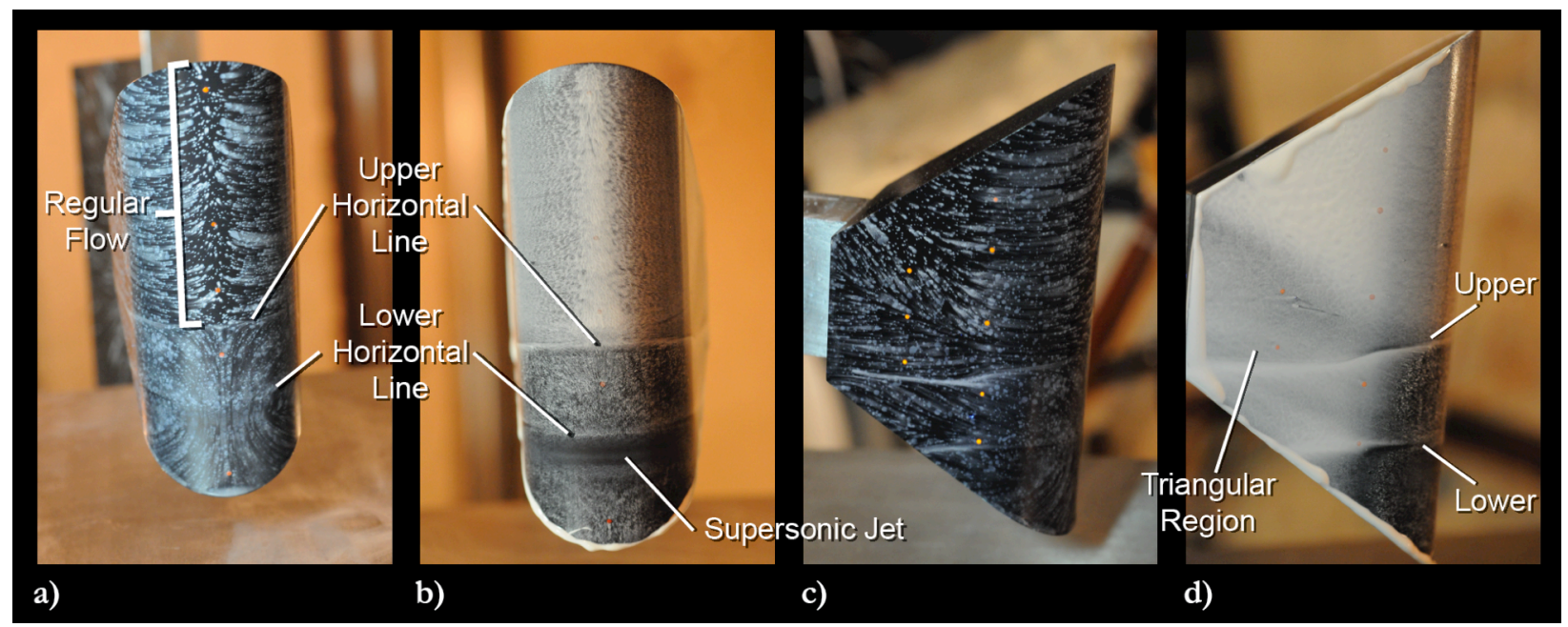

Figure 13. Oil-flow images of a Type IV interaction for the 0.75 -in. radius metal model at a $-15^{\circ}$ AoA for a) leading-edge view, dots, b) leading-edge view, full coating, c) side view, dots, d) side view, full coating.

The contour maps in Fig. 14 and Fig. 15 correspond to a Type IV interaction on the 0.25-, 0.50- and 0.75-in. radius models, from left to right, at a $-15^{\circ}$ AoA. The shock interaction wraps around the model, creating streaks of higher heating coefficients on the side. A narrow peak (shown in pink with heating values either at or greater than the maximum limit on the color bar) on the leading edge corresponds to the supersonic jet impingement point. This peak region widens as the leading-edge radius increases. The higher heating regions above and below the peak region also spread farther parallel to (and around the circumference of) the leading edge as the radius increases. 


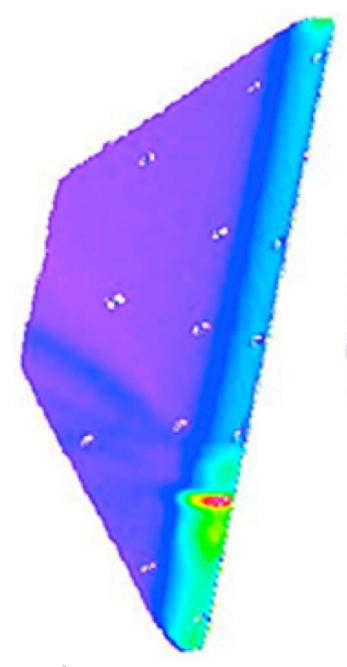

a)

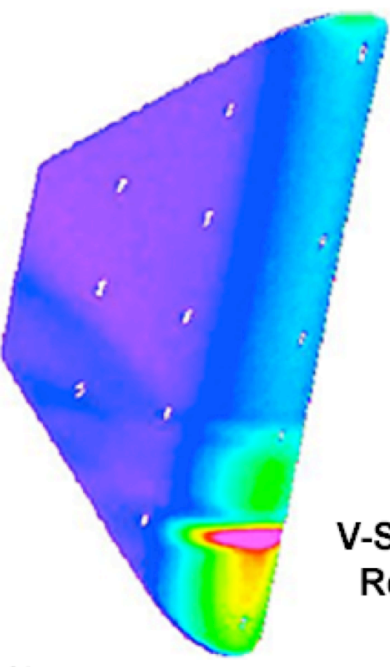

b)

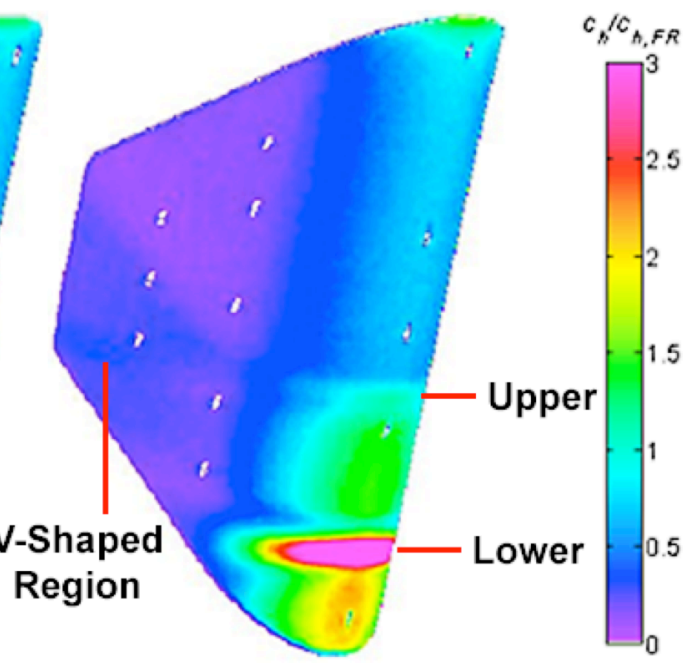

c)

Figure 14. IHEAT $c_{h} / c_{h, F R}$ contour maps of a Type IV interaction for a) 0.25-, b) 0.50-, and c) 0.75 -in. radius models at a $-15^{\circ} \mathrm{AoA}$.

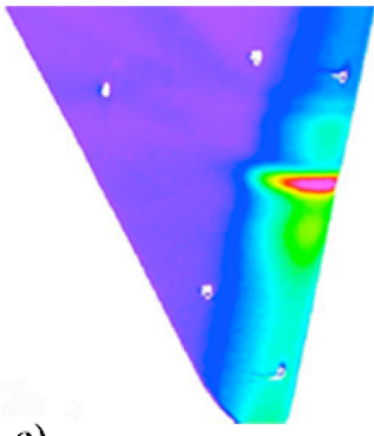

a)

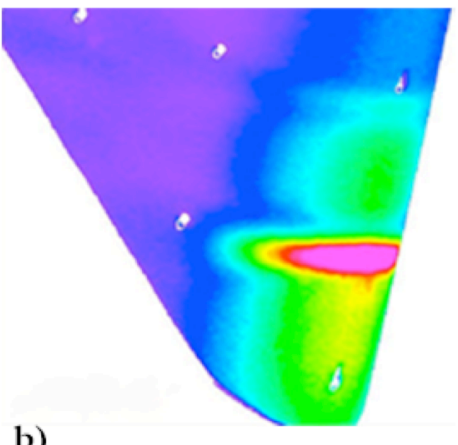

b)

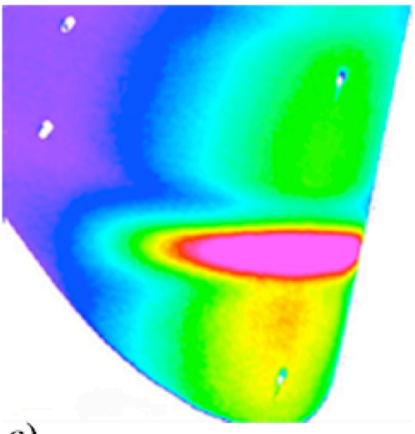

c)

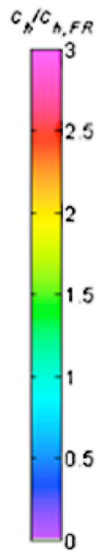

Figure 15. IHEAT $c_{h} / c_{h, F R}$ contour maps of a Type IV interaction for a) 0.25-, b) 0.50-, and c) 0.75-in. radius models at a $-15^{\circ}$ AoA (zoomed in).

The direct Type IV shock-shock interaction produces a sharp, narrow peak heat transfer augmentation as shown in Fig. 16. Peak values were derived from the zoomed-in data for this case since the few data points in the zoomedout peak region for the 0.25 -in. radius model exceed the phosphor limit by the time $t=1.8 \mathrm{~s}$. The peak has a maximum magnitude of 4.6 for the smallest model and about 6.8 for the largest model located at approximately $x / L_{0.25}=0.24$. The width of the peak region ranges from approximately 0.025 non-dimensionally (or $0.1 \mathrm{in}$.) to 0.06 (or 0.24 in.). Two small peaks exist on either side of the peak heat transfer coefficient for the smallest model. The unsteady vortex above the incident shock, likely combined with lateral conduction effects, yields a region of increased heating bounded by the upper and lower marks in Fig 14c) with either a smaller peak of about 1.5 for the smallest model or a broader peak of about $c_{h} / c_{h, \text { ref }}=2$ for the larger models.

Since the flow that contacts the model surface between $x / L_{0.25}=0$ and 0.2 passes through both the incident shock and the bow shock, the heat transfer coefficients below the major peak along the leading edge of the model exceed the baseline value with a small plateau of heat transfer coefficients between 2.3 and 2.9. The increased heating at the upper tip of the model is evident in plot a) of Fig. 16. The shock-shock interaction moves down the leading edge as the radius of the model increases, affecting the heat transfer to the lower tip of the model. The heat transfer coefficient contours near the lowest fiducial on the leading edge change from green to yellow and orange as the model radius increases. The shorter the distance between the peak and the edge of the model is, the greater the heat flux is in that region due to conduction from the peak down the length of the model. 


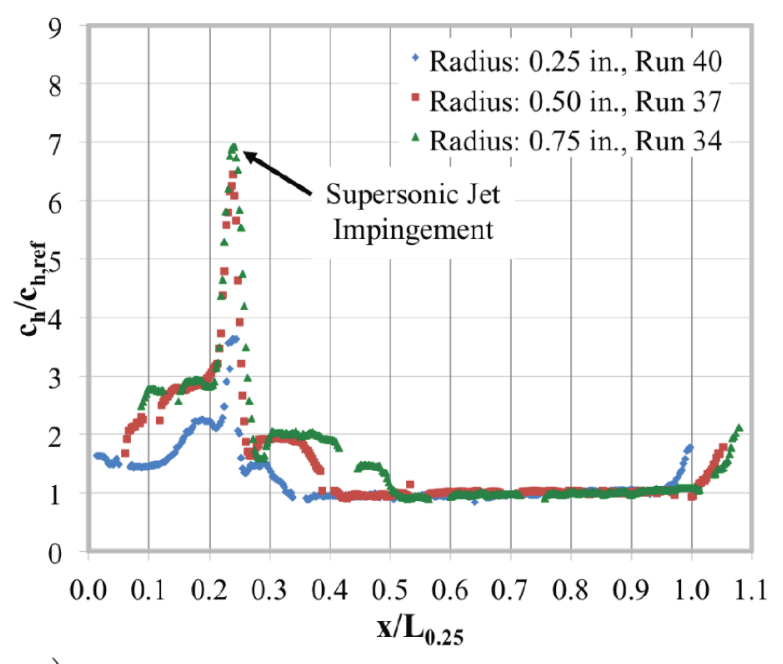

a)

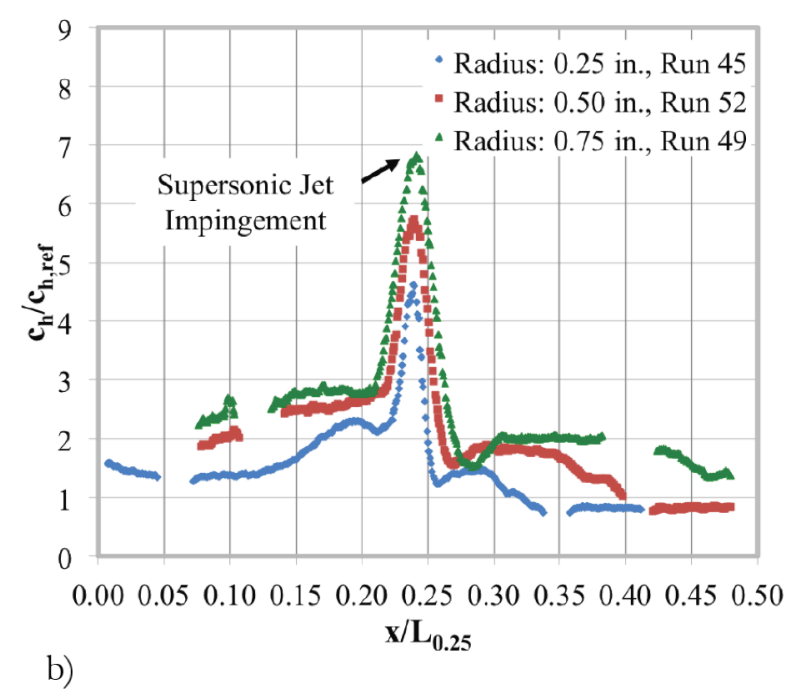

Figure 16. Non-dimensional heat transfer coefficients at $t=1.8 \mathrm{~s}$ from the IHEAT code for a Type IV interaction for the three fused silica models at a $-15^{\circ} \mathrm{AoA}$ for a) zoomed-out and b) zoomed-in data.

\section{Fin sweep of $-25^{\circ}$}

Figure 17 shows schlieren images (from left to right) of a Type III interaction for the $0.25-, 0.50$ - and 0.75 -in. radius models at a $-25^{\circ} \mathrm{AoA}$. In the Type III interaction, the supersonic jet of the previous two interaction types is replaced with a shear layer that attaches to the surface of the model. Supersonic flow exists in the triangular region between the turned bow shock and the shear layer in the image. ${ }^{1}$ Although the shear layer attachment point is not clearly evident in the zoom schlieren images, the shear layer leaves the triple point with the incident and bow shocks at the appropriate angle to connect to the model surface at the same location as the reflected shock. An unsteady region between the model surface and the shear layer near the attachment point is labeled as a density gradient in Fig. 17b). This phenomenon is clearer in schlieren videos of the interaction for the three geometries and contributes to the difficulty in capturing the shear layer attachment in a still image. As with the $-15^{\circ}$ AoA case, the interaction impingement point moves down the leading edge of the model as the leading-edge radius increases.

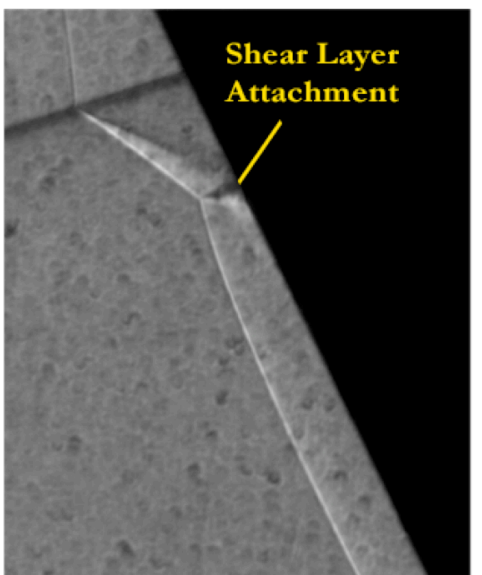

a)

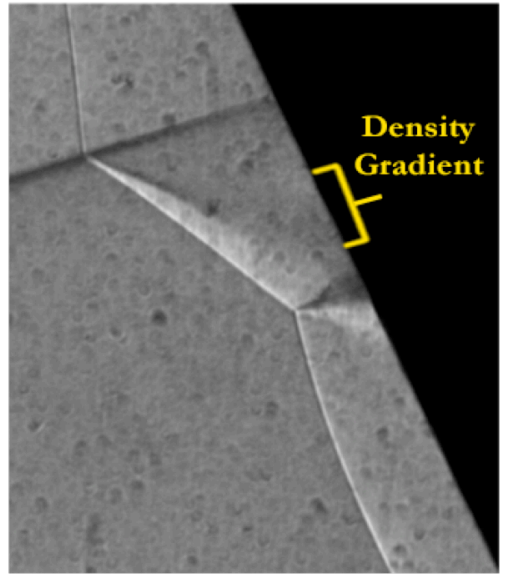

b)

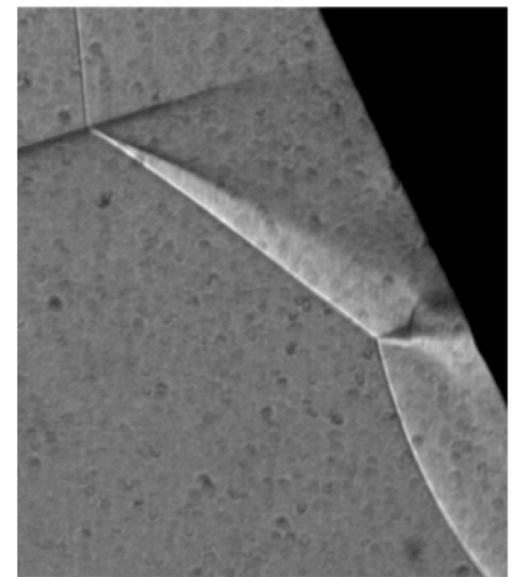

c)

Figure 17. Zoom schlieren images of a Type III interaction for a) 0.25-, b) 0.50-, and c) 0.75-in. radius models at a $-25^{\circ}$ AoA.

The Type III shock-shock interaction mainly affects the leading-edge streamlines near the extreme lower tip of the model as shown in Fig. 18 for the 0.75 -in. radius model. The streamline behavior for this model is similar to the patterns observed on the 0.25 - and 0.50 -in. radius models, although the shock-interaction region occurs higher on the leading edge in those cases. The pattern in the interaction region also resembles the oil flow streamlines obtained in a Type III interaction for a 1.18-in. wide flat plate in Ref. 1 . As in the $-15^{\circ}$ AoA oil flow images, the streamlines 
near the top of the leading edge in both images follow a curved path down and away from the stagnation region. The streamlines for a $-25^{\circ}$ AoA move farther downward than for the $-15^{\circ}$ case, as expected, since the bow shock is angled farther forward and the flow crossing the shock turns down at a sharper angle.

The shock-interaction region produces a stagnation region below the shear layer attachment point from which the streamlines on the leading edge fan out as shown in image a) obtained using dots of oil. The motion of the flow above and below the shear layer impingement location as demonstrated in the zoom schlieren data agrees with the orientation of these streamlines. The c) and d) oil flow images in Fig. 18 were captured at an angle rather than horizontally. Thus, the line wrapping around the images near the extrapolated "incident shock" location appears to be angled downward but instead should be roughly horizontal.

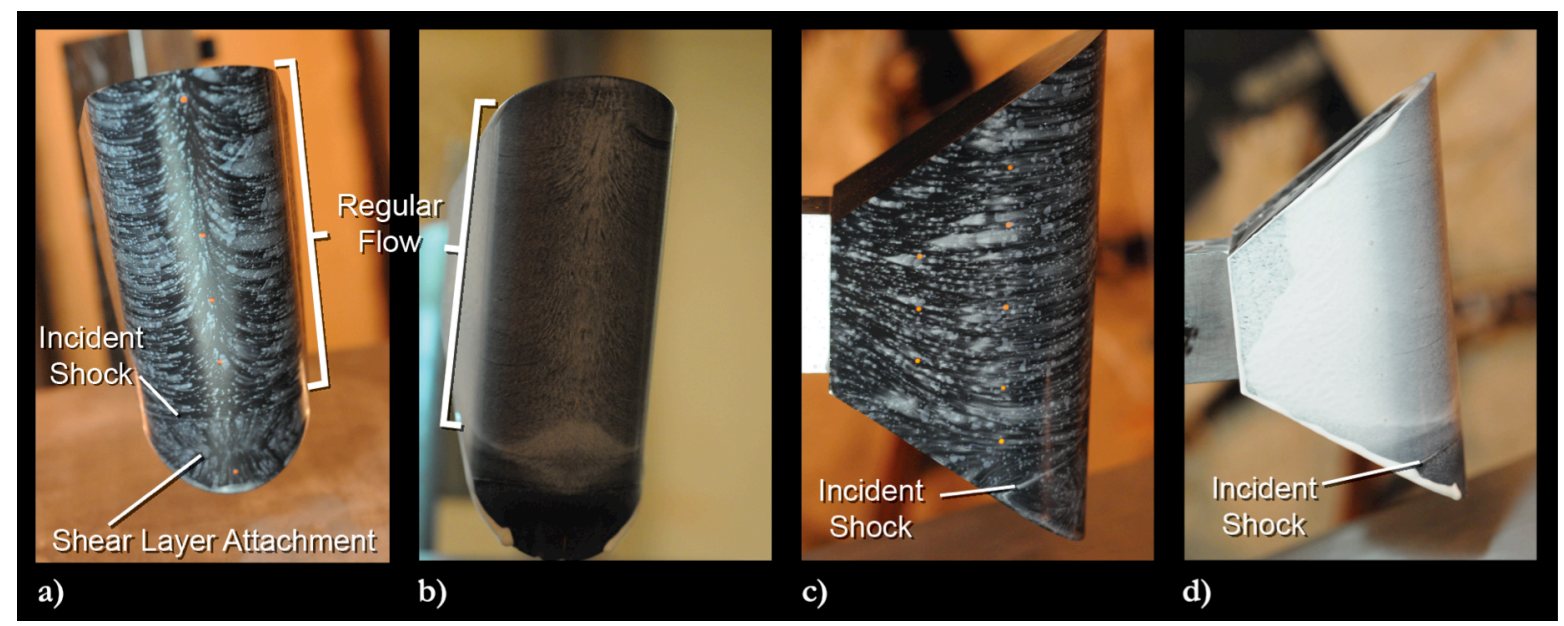

Figure 18. Oil-flow images of a Type III interaction for the 0.75 -in. radius metal model at a $-25^{\circ} \mathrm{AoA}$ for a) leading-edge view, dots, b) leading-edge view, full coating, c) side view, dots, d) side view, full coating.

A Type III shock-shock interaction produces a broader peak heat transfer. Contour maps for the $0.25-, 0.50$ - and 0.75-in. radius models (from left to right) at a $-25^{\circ}$ AoA are included in Fig. 19 and Fig. 20. The effects of the shock interaction wrap around the model, creating streaks of higher heating. Again, the width of the peak heat transfer region increases laterally and circumferentially with increasing leading-edge radius. The extrapolated incident shock location is near a green ellipse above the main peak that leads to a blue line of higher heat transfer values around the side of the model. This ellipse of elevated heating is likely due to the density gradient below the incident shock in the schlieren images and videos, which may be caused by the stagnation of the opposing streamlines along the leading edge. Videos generated from numerical schlieren images in Ref. 15 demonstrate the flow due to the attaching shear layer moves up and the flow passing through the bow shock above the interaction moves down.

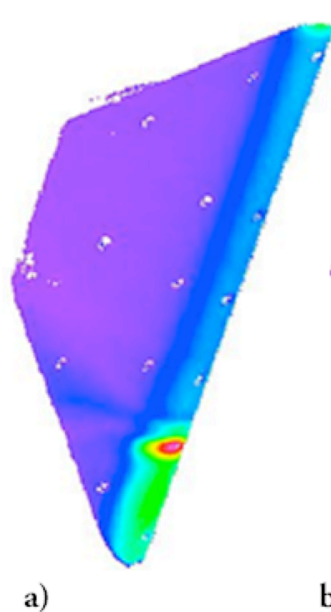

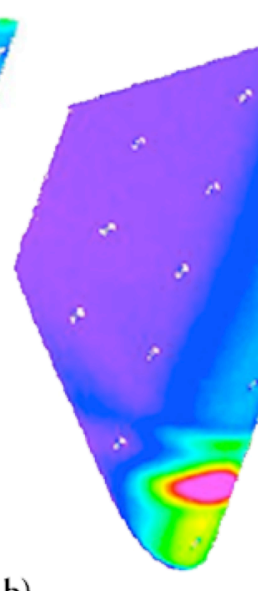

b)

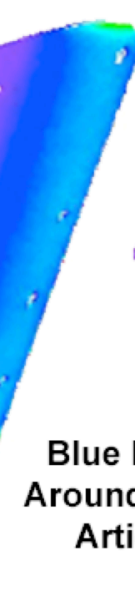

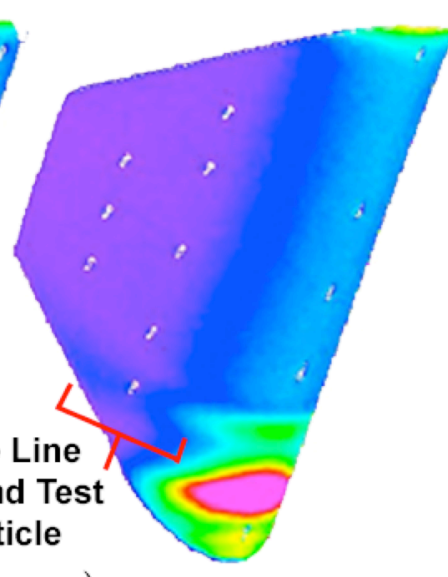

c)

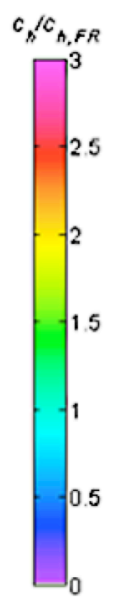

Figure 19. IHEAT $c_{h} / c_{h, F R}$ contour maps of a Type III interaction for a) 0.25-, b) 0.50-, and c) 0.75-in. radius models at a $-25^{\circ} \mathrm{AoA}$. 


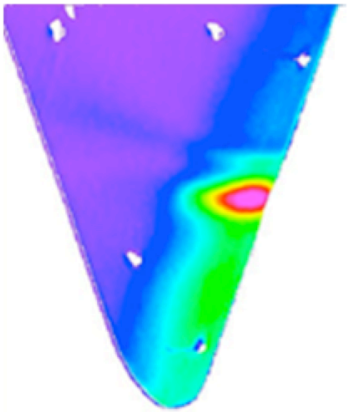

a)

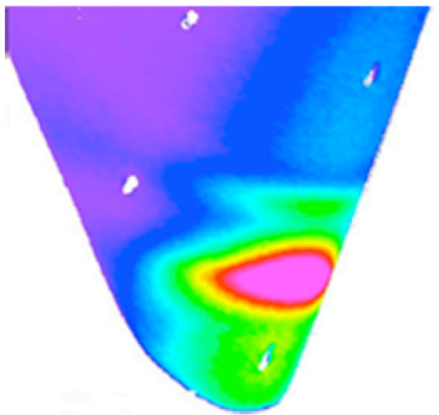

b)

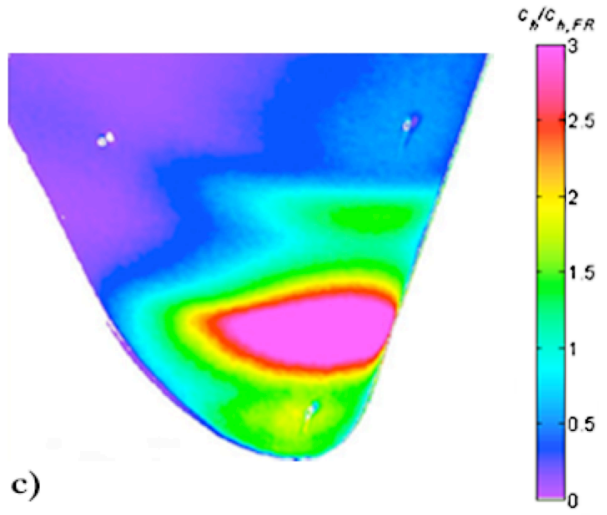

Figure 20. IHEAT $c_{h} / c_{h, F R}$ contour map of a Type III interaction for a) 0.25-, b) 0.50-, and c) 0.75-in. radius models at a $-25^{\circ}$ AoA (zoomed in).

The zoomed-in plot in Fig. 21 shows a peak at the shear layer attachment point $\left(\right.$ at $\left.x / L_{0.25}=0.22\right)$ with a width of 0.065 ( $0.26 \mathrm{in}$.) and a maximum value of at least 5.2 for the 0.25 -in. radius model. Again, the actual peak is not calculated for the zoomed-out line cut in this case since the maximum temperature exceeds the phosphor limit at $t=$ $1.8 \mathrm{~s}$. Both the width and the maximum value of the peak due to the shear layer attachment point increase to 0.14 non-dimensionally (or 0.55 in.) and 7.9, respectively, for the 0.75 -in. radius model.

The heat transfer to the lower portion of the leading edge is about twice the baseline heating for the smallest model, and higher for the larger models. Warmer air that passed through the bow and incident shock may be partially responsible for this heating, as well as conduction through the model and the proximity of the higher peak relative to the tip of the model. Since the angle of the shear layer relative to the incident shock for a Type III interaction varies by a few degrees between the three model geometries, the shear layer attachment point moves down the leading edge as the bow shock standoff distance increases and produces a greater distance between the triple point and the surface of the model. Therefore, the region of peak heating moves closer to the lowest fiducial mark as the leading-edge radius increases for a Type III interaction, as expected from the zoom schlieren images. The line cuts in Fig. 21 are translated to align the heating peaks for the different models.
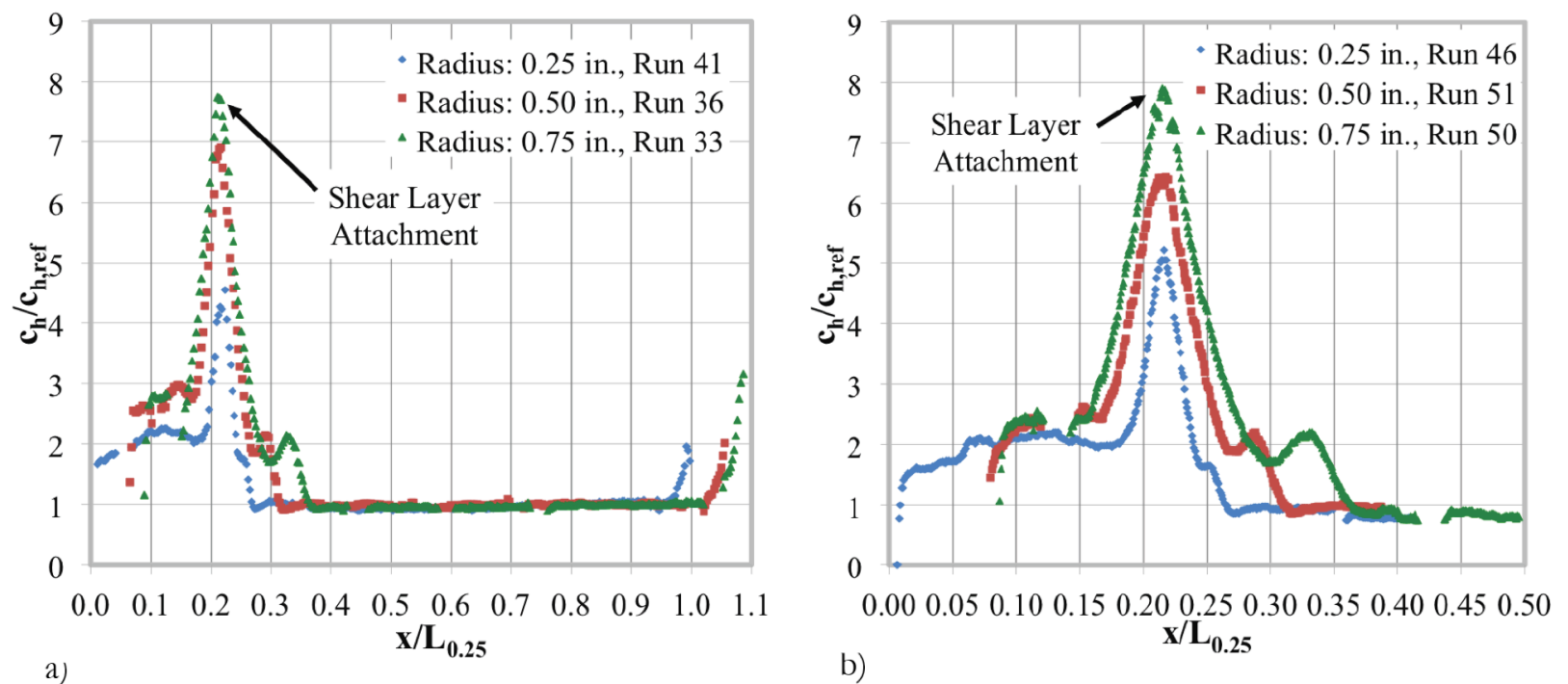

Figure 21. Non-dimensional heat transfer coefficients at $t=1.8 \mathrm{~s}$ from the IHEAT code for a Type III interaction for the three fused silica models at a $-25^{\circ}$ AoA for a) zoomed-out and b) zoomed-in data.

Additional information about the magnitude and location of the peaks and valleys in the line cuts is in Ref. 15 . For example, a unit $\mathrm{Re}_{\infty}$ number sweep was conducted to determine the impact of the unit $\mathrm{Re}_{\infty}$ number on the peak heating due to the Type IV interaction. Also, 1D FV data is provided to compare to the IHEAT results for each case. 


\section{1. $1 D$ versus $2 D$ Heat Transfer}

Temperature data obtained in a run with a flow unit $\mathrm{Re}_{\infty}$ number of $1.1 \times 10^{6} / \mathrm{ft}$ was used to compute leadingedge heat transfer coefficients to provide a comparison between 1D and 2D heat transfer analyses. The plots in Fig. 22 and Fig. 23 show enthalpy-based a) dimensional and b) non-dimensional heat transfer coefficients along the leading edge of a 0.25 -in. radius model at a $-15^{\circ} \mathrm{AoA}$. The input data to the FV codes for this run was changed from every frame to every sixth frame ( $0.2 \mathrm{~s}$ apart) of the recorded surface temperature data obtained during Run 43 . The radial spacing is $\Delta r=2.5 \times 10^{-4}$ in. between nodes. Data is extracted from 358 pixels along the leading edge, so the lateral spacing is $\Delta z=1.12 \times 10^{-2}$ in. between nodes. The maximum value of the y-axis of the dimensional plot in Fig. 22 is 0.40 and in Fig. 23 is 0.30 since these two plots show coefficients in units of $\mathrm{lb}_{\mathrm{m}} / \mathrm{ft}^{2}$-s. The limits on the $\mathrm{x}$-axis were also changed to $\mathrm{x} / \mathrm{L}=0.1$ to 0.5 for the plots in these two figures to better show the differences between the 1D and 2D line cut data. The full line cuts for these comparisons are presented in Ref. 15.

The dimensional heat transfer coefficients derived using a 2D method in the region away from the shock-shock interaction region on the leading edge are lower than those calculated by either $1 \mathrm{D}$ method. This trend is correct since the 2D code accounts for lateral conduction, thereby reducing the heat assumed to travel in the radial direction since heat also moves to either side of the cell in the lateral direction. The peak heating augmentation due to the supersonic jet impingement is narrower and higher for the $2 \mathrm{D}$ case than for the $1 \mathrm{D}$ cases. For the dimensional line cuts in Fig. 22, at $t=3.4 \mathrm{~s}$ into the run, the 2D FV peak heat transfer is about $16 \%$ greater than the peak value from IHEAT. The 2D FV non-dimensional peak heat transfer coefficient is approximately $38 \%$ higher than the corresponding IHEAT peak value. The non-dimensional coefficients away from the peak collapse on top of each other, suggesting the offset from the IHEAT output due to the 1D and 2D FV methods is uniform in those regions.

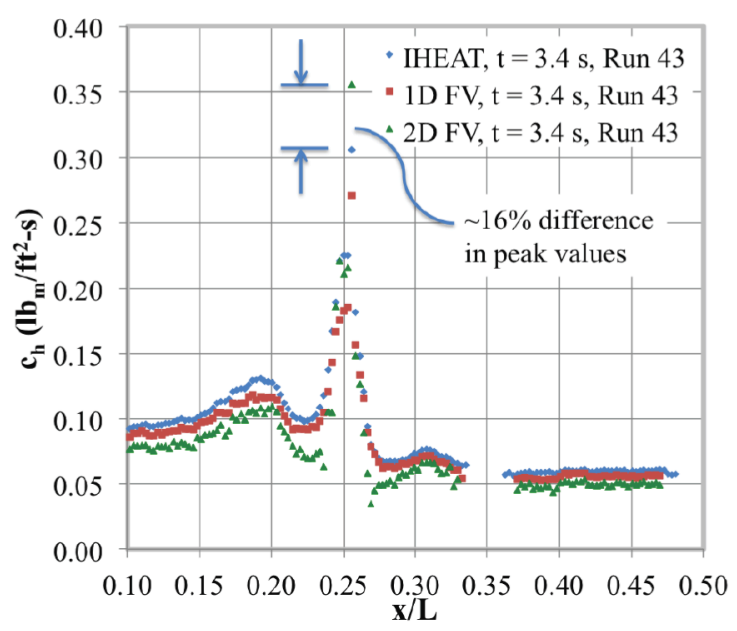

a)

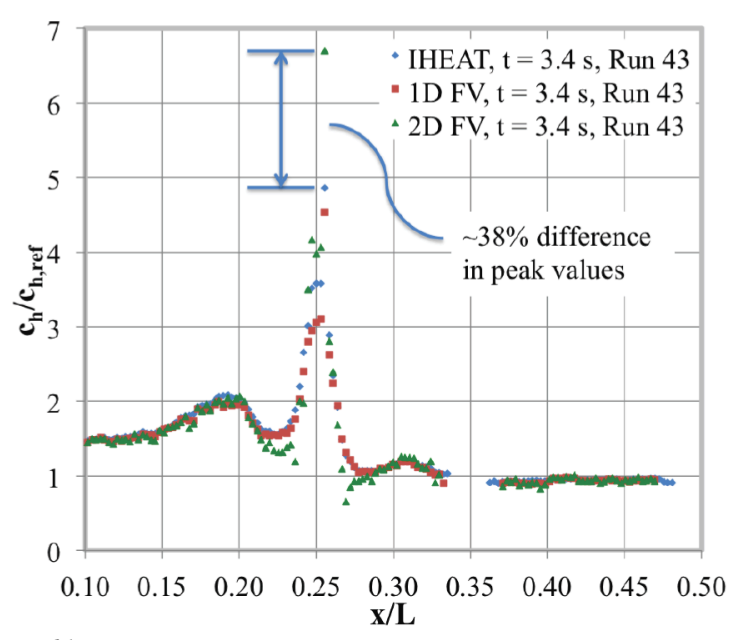

b)

Figure 22. a) Dimensional and b) non-dimensional heat transfer coefficients from the IHEAT, 1D FV, and 2D FV codes for a Type IV interaction for the 0.25-in. radius model at a $-15^{\circ} \mathrm{AoA}$ and a unit $\mathrm{Re}_{\infty}=1.1 \times 10^{6} / \mathrm{ft}$

In the dimensional plot in Fig. 23, the 2D peak heat transfer is about $20 \%$ greater than the IHEAT result. As expected, the difference between the IHEAT and 2D outputs increases later in the run due to errors associated with neglecting the lateral conduction and the semi-infinite assumption in IHEAT. Again, the difference between the heat transfer peaks for the 2D and IHEAT codes increases when the heat transfer coefficients are divided by a reference value to yield non-dimensional data. In the non-dimensional plot, the $2 \mathrm{D} \mathrm{FV}$ peak heat transfer augmentation is about $47 \%$ higher than the IHEAT peak value. Berry and Nowak ${ }^{11}$ predicted a non-dimensional peak heating augmentation in the Type IV interaction produced by a $-15^{\circ}$ AoA for a unit $\operatorname{Re}_{\infty}=2.1 \times 10^{6} / \mathrm{ft}$ would increase $43 \%$, from about 7 to 10 , if lateral conduction effects were considered in the heat transfer analysis.

Temperature data is also available along the entire leading edge near the end of the wind tunnel runs for the lower heating cases. Thus, the $2 \mathrm{D} \mathrm{FV}$ code was implemented using the temperature data at $t=4.6 \mathrm{~s}$ for the 0.75 -in. radius model at a $0^{\circ} \mathrm{AoA}$ to compare to the 1D IHEAT results. This comparison is shown for non-dimensional heat transfer coefficients in Fig. 24. The greatest difference between the 1D and 2D results is at the valley between the extrapolated incident shock location and the shear layer attachment points. In the non-dimensional heat transfer coefficients, the 2D FV value for the minimum heat transfer coefficient in this valley is about $12 \%$ lower than the minimum IHEAT value. The non-dimensional $1 \mathrm{D}$ and 2D heat transfer coefficients are similar away from the valley. 


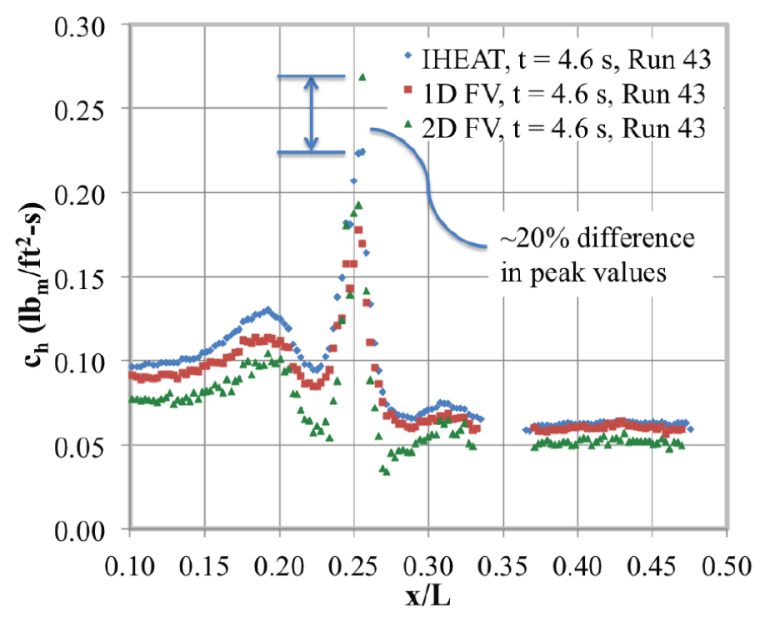

a)

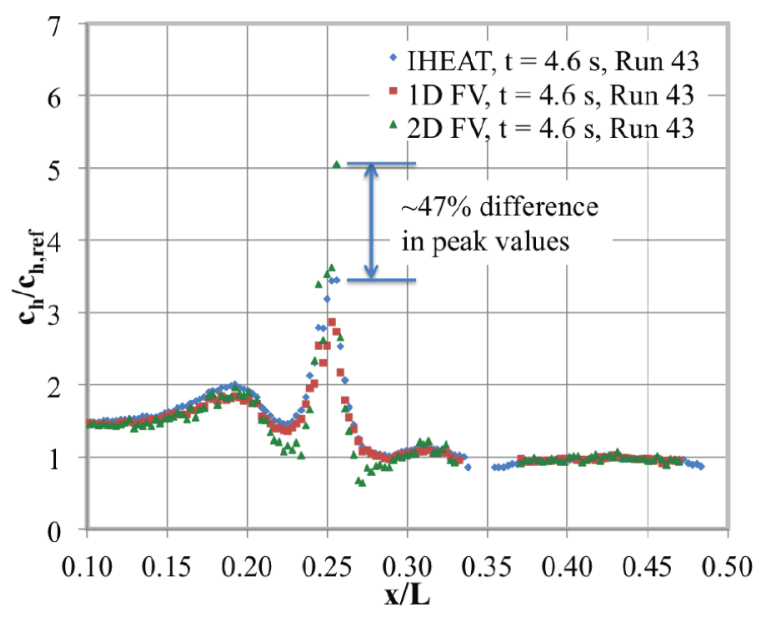

b)

Figure 23. a) Dimensional and b) non-dimensional heat transfer coefficients from the IHEAT, 1D FV, and 2D FV codes for a Type IV interaction for the 0.25 -in. radius model at a $-15^{\circ} \mathrm{AoA}$ and a unit $\mathrm{Re}_{\infty}=1.1 \times 10^{6} / \mathrm{ft}$.

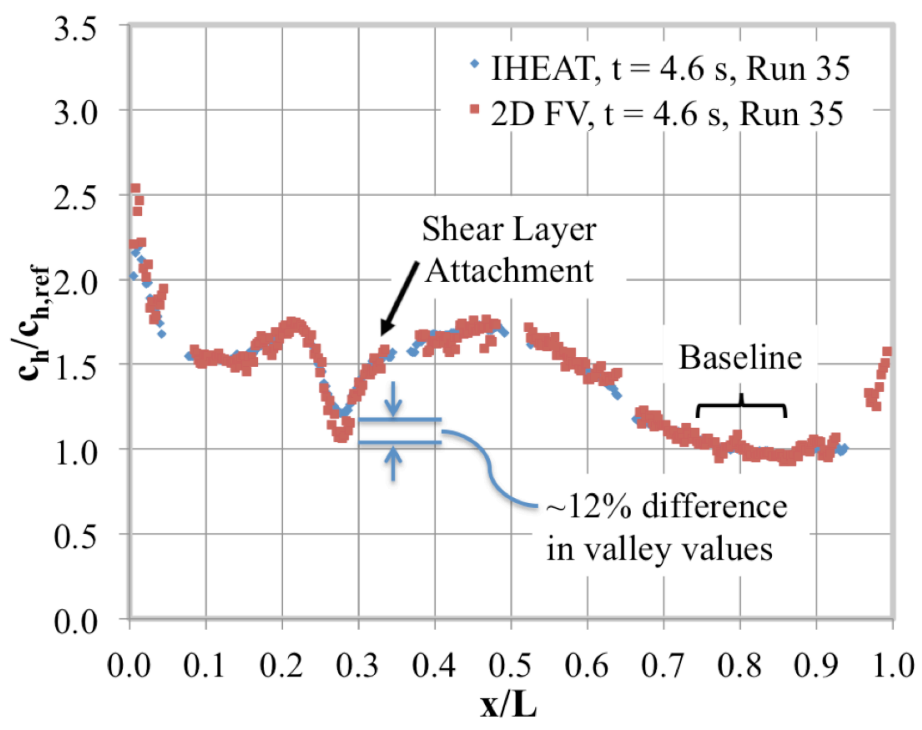

Figure 24. Non-dimensional heat transfer coefficients at $t=4.6 \mathrm{~s}$ from the IHEAT and 2D FV codes for a Type IVa interaction for the 0.75-in. radius model at a $0^{\circ} \mathrm{AoA}$ and a unit $\operatorname{Re}_{\infty}=2.1 \times 10^{6} / \mathrm{ft}$.

\section{Spatial Resolution}

Separate runs were conducted for each model configuration with the phosphor camera either zoomed out to capture the full side and leading edge of the model or zoomed in to view only the areas near the shock-shock interaction. The plot in Fig. 25 shows a comparison of the peak region between the non-dimensional heat transfer coefficients for the zoomed-in and zoomed-out runs with a $-15^{\circ}$ AoA and a unit $\mathrm{Re}_{\infty}=1.1 \mathrm{x} 10^{6} / \mathrm{ft}$. The $\mathrm{x}$-axis positions for the zoomed-in data were shifted by a small amount to align the peak location between the two runs.

The spatial resolution of the zoomed-in data is about 3.8 times greater than the resolution for the zoomed-out heat transfer data. The spatial resolution of the phosphor data ranged from $0.0105 \mathrm{in}$. to $0.015 \mathrm{in}$. between the acquired temperatures for the zoomed-out cases, and the resolution was about $0.004 \mathrm{in}$. for the zoomed-in cases (or 0.0034 in. for the configuration in Fig. 25). These spatial resolutions are sufficient for the heat transfer experiments and confirm the minimum thin-film-gage spacing of 0.015 in. found in Ref. 11 deemed necessary to capture the narrow heating spike for Type III and IV interactions accurately. Thus, the increased resolution of the zoomed-in data provides additional confidence that the observed trends in the zoomed-out data properly represent the heat transfer behavior for each shock-shock interaction. 


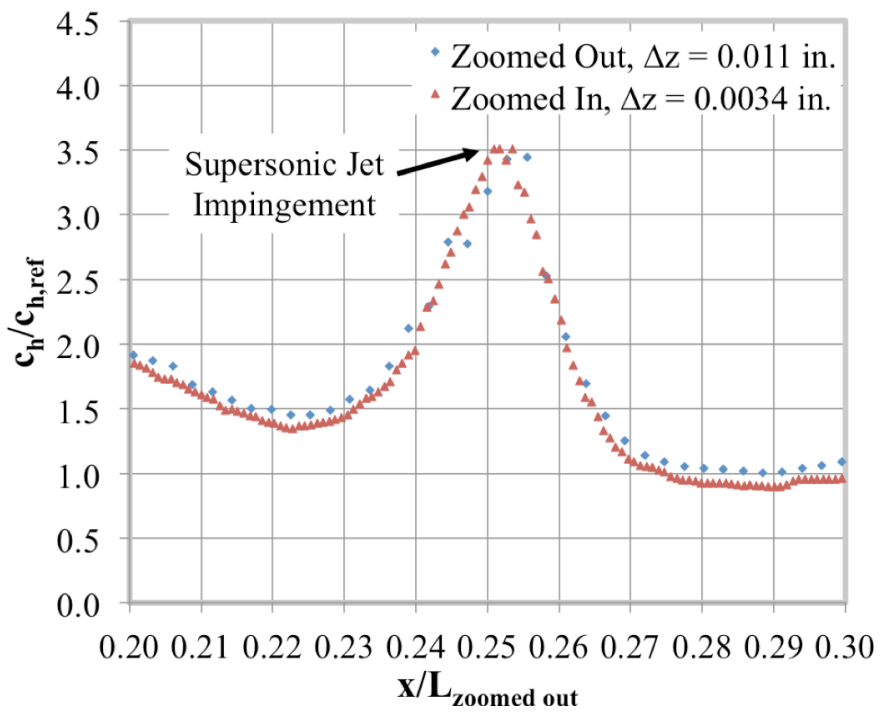

Figure 25. Spatial resolution of the IHEAT zoomed-in versus zoomed-out non-dimensional peak heat transfer coefficients at $t=4.6 \mathrm{~s}$ for a Type IV interaction for the 0.25 -in. radius model at a $-15^{\circ} \mathrm{AoA}$ and a unit $\operatorname{Re}_{\infty}=1.1 \times 10^{6} / \mathrm{ft}$.

\section{Conclusions}

The Type IVa $\left(0^{\circ} \mathrm{AoA}\right)$, direct Type IV $\left(-15^{\circ} \mathrm{AoA}\right)$, and Type III $\left(-25^{\circ} \mathrm{AoA}\right)$ shock-shock interactions were investigated using three model geometries with leading-edge radii of $0.25 \mathrm{in}$., $0.50 \mathrm{in}$., and 0.75 in., respectively. Flow density gradients were observed using a zoom schlieren technique and the surface flow was visualized using oil flow methods. The general heating behavior for these three interactions were assessed using 1D IHEAT contour maps. The leading-edge heat transfer coefficients were analyzed for each run using 1D semi-infinite and finitevolume methods. A 2D finite-volume method was also used to analyze the heat transfer behavior for two cases.

Walker and $\operatorname{Scott}^{22}$ and Wright et al. ${ }^{23}$ recommend a greater spatial resolution than 0.015 in. between gages to experimentally resolve the heat transfer peak for the Type IV interaction. The spatial resolution of the phosphor thermography data ranges from $0.004 \mathrm{in}$. to $0.015 \mathrm{in}$. Therefore, the data resolution is better than or comparable to the discrete temperature gage spacing on models utilized in previous shock-shock interaction studies. This improved spatial resolution is significant because the heat transfer data from these line cuts display similar trends to equivalent cases in Ref. 11. Thus, the assertion in Ref. 11 that 0.015 -in. gage spacing is sufficient to accurately capture the narrow peak heat transfer augmentation due to a Type IV interaction is confirmed. The zoomed-in line cut data yielded lower than expected results for the non-dimensional peak heat transfer data in several cases, likely due to the limitation on the reference values used to convert the heat transfer data for these runs to non-dimensional values.

Based on these observations, the Type IVa shock-shock interaction exhibits the smallest peak heating augmentation. The Type IV interaction produces a narrow, steep heat transfer peak due to an impinging supersonic jet. The Type III interaction does not include a supersonic jet as in the other two cases, but instead yields a broader peak in the heat transfer coefficients at the shear layer attachment point. Qualitatively, the heat transfer profiles from this study are similar to the corresponding Type III and IV cases in Ref. 11.

The non-dimensional peak heat transfer for a given type of shock-shock interaction increases as the leadingedge radius increases, and the dimensional heat transfer coefficients follow the opposite trend. As the leading-edge radius increases, the baseline heat transfer coefficients away from the shock-shock interaction region decrease. This trend yields a lower averaged reference value for the larger models, which amplifies the non-dimensional peak heat transfer augmentation compared to the smaller model geometries.

Dimensionally, the peak heat transfer coefficient from a 2D finite-volume analysis was approximately $20 \%$ higher than the result from a 1D semi-infinite analysis for a Type IV interaction, which corroborates the findings in Ref. 22 for a shock-shock interaction case and in Ref. 24 for striation heating due to streams of injected gas. The non-dimensional peak heat transfer augmentation calculated for a Type IV interaction at a unit $\mathrm{Re}_{\infty}=1.1 \times 10^{6} / \mathrm{ft}$ using a $2 \mathrm{D}$ ADI method is about $47 \%$ higher than the value predicted using the $1 \mathrm{D}$ semi-infinite IHEAT code at a time $4.6 \mathrm{~s}$ into the run. Berry and Nowak ${ }^{11}$ predicted a similar non-dimensional peak heating augmentation for a 
case with a unit $\operatorname{Re}_{\infty}=2.1 \times 10^{6} / \mathrm{ft}$ would increase $43 \%$ from about 7 to 10 if lateral conduction effects were considered in the heat transfer analysis. This difference between $1 \mathrm{D}$ and $2 \mathrm{D}$ heat transfer results is a reasonable estimate if the results for the unit $\mathrm{Re}_{\infty}=1.1 \times 10^{6} / \mathrm{ft}$ case can be extrapolated to a unit $\mathrm{Re}_{\infty}=2.1 \times 10^{6} / \mathrm{ft}$ case. For the Type IVa interaction with smaller temperature gradients in the lateral direction, the difference between the 1D and 2D heat transfer results was likewise smaller. Thus, a multi-dimensional conduction analysis is necessary to account for lateral conduction in cases with large temperature gradients.

\section{Acknowledgments}

The authors would like to thank Kamran Daryabeigi for his help in writing and debugging the finite volume conduction codes used in this study. The authors also thank Elaine Scott, Michael von Spakovsky, Walter O’Brien, and Shann Rufer for their assistance in this research.

\section{References}

1 Edney, Barry. Anomalous Heat Transfer and Pressure Distributions on Blunt Bodies at Hypersonic Speeds in the Presence of an Impinging Shock. The Aeronautical Research Institute of Sweden, Stockholm, 1968.

2 Andreadis, Dean. Scramjet Engines Enabling the Seamless Integration of Air \& Space Operations. Pratt \& Whitney, West Palm Beach, FL.

3 Harwood, William. Board Hearing Shows Work to Piece Together Known Data. Spaceflight Now Inc. [online resource]. [updated Mar 2003, cited Mar 2013]. URL: http://www.spaceflightnow.com/shuttle/sts107/030318hearing/.

4 Watts, Joe D. Flight Experiment with Shock Impingement and Interference Heating on the X-15-2 Research Airplane. Washington D.C., 1968.

5 Keyes, J. W. and Hains, F. D. Analytical and Experimental Studies of Shock Interference Heating in Hypersonic Flows. Washington D.C., May 1973.

6 Nowak, R., Holden, M., and Wieting, A. Shock/shock interference on a transpiration cooled hemispherical model. (Seattle, WA June 1990), AIAA 90-1643.

7 Bushnell, Dennis M. Interference Heating on a Swept Cylinder in Region of Intersection with a Wedge at Mach Number 8. NASA, Washington, D.C., 1965.

8 Bushnell, Dennis M. Effects of Shock Impingement and Other Factors on Leading-Edge Heat Transfer. NASA, Washington, D.C., April 1968.

9 Carter, Howard S. and Carr, Robert E. Free-Flight Investigation of Heat Transfer to an Unswept Cylinder Subjected to an Incident Shock and Flow Interference from an Upstream Body at Mach Numbers up to 5.50. NASA Langley Research Center, Washington, D.C., 1961.

${ }^{10}$ Hiers, Robert S. and Loubsky, William J. Effects of Shock-Wave Impingement on the Heat Transfer on a Cylindrical Leading Edge. NASA, Washington, D.C., 1967.

${ }^{11}$ Berry, Scott A. and Nowak, Robert J. Fin Leading-Edge Sweep Effect on Shock-Shock Interaction at Mach 6. Journal of Spacecraft and Rockets, 34, 4 (July-August 1997), 416-425.

${ }^{12}$ Berger, Karen T., Hollingsworth, Kevin E., Wright, Sheila A., and Rufer, Shann J. NASA Langley Aerothermodynamics Laboratory: Hypersonic Testing Capabilities. (Kissimmee, FL 2015), AIAA 2015-1337.

${ }^{13}$ Rhode, Matthew N. and DeLoach, Richard. Hypersonic Wind Tunnel Calibration Using the Modern Design of Experiments. (Tucson, AZ 2005), AIAA 2005-4274.

${ }^{14}$ Hollis, Brian R. and Collier, Arnold S. Turbulent Aeroheating Testing of Mars Science Laboratory Entry Vehicle. Journal of Spacecraft and Rockets, 45, 3 (2008), 417-427.

${ }^{15}$ Jones, Michelle L. Experimental Investigation of Shock-Shock Interactions over a 2-D Wedge at M=6. Virginia Polytechnic Instititute and State University, Hampton, VA, 2013, Master's Thesis.

${ }^{16}$ Buck, G. M., Powers, M. A., Griffith, M. S., Hopkins, J. W., Veneris, P. H., and Kuykendoll, K. A. Fabrication of 0.0075-Scale Orbiter Phosphor Thermography Test Models for Shuttle RTF Aeroheating Studies. Hampton, Virginia, November 2006.

${ }^{17}$ Merski, N. R. Reduction and Analysis of Phosphor Thermography Data with the IHEAT Software Package. ( 1997), AIAA 1998-0712. 
${ }^{18}$ Merski, N. R. Global Aeroheating Wind-Tunnel Measurements Using Improved Two-Color Phosphor Thermography Method. Journal of Spacecraft and Rockets, 36, 2 (March-April 1999), 160-170.

${ }^{19}$ Burden, Richard L. and Faires, J. Douglas. Numerical Analysis: Eigth Edition. Thomson, Brooks/Cole, Belmont, CA, 2005.

${ }^{20}$ Tannehill, John C., Anderson, Dale A., and Pletcher, Richard H. Computational Fluid Mechanics and Heat Transfer, Second Edition. Taylor \& Francis, Philadelphia, PA, 1997.

${ }^{21}$ Fay, J. A. and Riddell, F. R. Theory of Stagnation Point Heat Transfer in Dissociated Air. Journal of the Aeronautical Sciences, 25, 2 (February 1958), 73-85, 121.

${ }^{22}$ Walker, D. Greg and Scott, Elaine P. The Effects of Lateral Conduction on Heat Flux Estimation from Surface Temperature Measurements. AIAA/A.S.M.E. Joint Thermophysics and Heat Transfer Conference, 3 (June 1998), 245-252.

${ }^{23}$ Wright, M. J., Nowak, R. J., Berry, S. A., Glass, C. E., and Candler, G. V. Numerical/Experimental Investigation of 3-D Swept Fin Shock Interactions. (Albuquerque, NM June 1998), AIAA 1998-2816.

${ }^{24}$ Daryabeigi, K., Berry, S. A., Horvath, T. J., and Nowak, R. J. Finite Volume Numerical Methods for Aeroheating Rate Calculations from Infrared Thermographic Data. Journal of Spacecraft and Rockets, 43, 1 (January-February 2006), 54-62. 